\title{
Profiling the Human Phosphoproteome to Estimate the True Extent of Protein Phosphorylation
}

\author{
Anton Kalyuzhnyy ${ }^{1,3}$, Patrick A. Eyers ${ }^{1}$, Claire E. Eyers ${ }^{1,2}$, Zhi Sun ${ }^{4}$, Eric W. Deutsch ${ }^{4}$ and \\ Andrew R. Jones ${ }^{1,3}$
}

\begin{abstract}
${ }^{1}$ Department of Biochemistry and Systems Biology, Institute of Systems, Molecular and Integrative Biology, University of Liverpool, Liverpool, UK

${ }^{2}$ Centre for Proteome Research, Department of Biochemistry and Systems Biology, Institute of Systems, Molecular and Integrative Biology University of Liverpool, Liverpool, UK

${ }^{3}$ Computational Biology Facility, Department of Biochemistry and Systems Biology, Institute of Systems, Molecular and Integrative Biology University of Liverpool, Liverpool, UK
\end{abstract}

${ }^{4}$ Institute for Systems Biology, Seattle, Washington 98109, United States

\begin{abstract}
Mass spectrometry-based phosphoproteomics allows large-scale generation of phosphorylation site data. However, analytical pipelines need to be carefully designed and optimised to minimise incorrect identification of phosphopeptide sequences or wrong localisation of phosphorylation sites within those peptides. Public databases such as PhosphoSitePlus (PSP) and PeptideAtlas (PA) compile results from published papers or openly available MS data, but to our knowledge, there is no database-level control for false discovery of sites, subsequently leading to the likely overestimation of true phosphosites. It is therefore difficult for researchers to assess which phosphosites are "real" and which are likely to be artefacts of data processing. By profiling the human phosphoproteome, we aimed to estimate the false discovery rate (FDR) of phosphosites based on available evidence in PSP and/or PA and predict a more realistic count of true phosphosites. We ranked sites into phosphorylation likelihood sets based on layers of accumulated evidence and then analysed them in terms of amino acid conservation across 100 species, sequence properties and functional annotations of associated proteins. We demonstrated significant differences between the sets and developed a method for independent phosphosite FDR estimation. Remarkably, we estimated a false discovery rate of $86.1 \%, 95.4 \%$ and $82.2 \%$ within sets of described phosphoserine (pSer), phosphothreonine (pThr) and phosphotyrosine (pTyr) sites respectively for which only a single piece of identification evidence is available (the vast majority of sites in PSP). Overall, we estimate that $\sim 56,000 \mathrm{Ser}, 10,000 \mathrm{Thr}$ and 12,000 Tyr phosphosites in the human proteome have truly been identified to date, based on evidence in PSP and/or PA, which is lower than most published estimates. Furthermore, our analysis estimated $~ 91,000 \mathrm{Ser}, 49,000 \mathrm{Thr}$ and 26,000 Tyr sites that are likely to represent false-positive phosphosite identifications. We conclude that researchers should be aware of the significant potential for false positive sites to be present in public databases and should evaluate the evidence behind the phosphosites used in their research.
\end{abstract}




\section{Introduction}

Protein phosphorylation is a fundamental post-translation modification (PTM) which regulates protein function and is very well-studied in relation to cell signalling pathways and disease (Cohen, 2001; Cohen, 2002; Goedert et al., 1992). Huge numbers of phosphorylated peptides and sites have been reported and characterized after isolation from human cells using approaches allied to tandem mass spectrometry (LCMS/MS) which have primarily focussed on the phosphorylation of serine (Ser), threonine (Thr) and tyrosine (Tyr) residues (Amanchy et al., 2005; Nousiainen et al., 2006; Olsen et al., 2006; Olsen et al., 2010; Sharma et al., 2014). However, large numbers of non-canonical phosphorylation sites have also been annotated on proteins fractionated from human cells (Hardman et al., 2019). This additional complexity highlights the ongoing requirement for careful, evidence-based phosphosite identification from mass spectrometric datasets.

Historically, the analysis of phosphorylation sites in proteins tended to be accurate, initially relying on chromatography and solid-state Edman sequencing (Alessi et al., 1996; Wettenhall, Aebersold and Hood, 1991; Wettenhall, Erikson and Maller, 1992). However, such low-throughput approaches are now very rare, lacking the depth of coverage needed in modern proteomic workflows. The dominance of MS approaches has led to the development of multiple strategies to both understand and help mitigate the high levels of phosphopeptide false discovery rate (FDR), particularly in sets of mapped peptide spectral matches (PSMs) that result from LC-MS/MS and sequence database analysis (Elias and Gygi, 2007; Käll, Storey and Noble, 2009; Söderholm et al., 2014). The goal of such approaches is to separate true identifications from false ones. Even without considering non-canonical phosphorylation (which is likely not detectable in standard phosphoproteomics pipelines), many confidently-identified phosphopeptides possess multiple Ser, Thr or Tyr residues that could explain the number of phosphosites in a given proteolytically-generated peptide (Hardman et al., 2019). As such, statistical processes are applied, either within the search engine used for peptide mapping, or in a downstream software application to calculate additional statistics, such as a local false localisation rate (FLR) or conversely the probability that a given site within a peptide is correct or incorrect. Software / algorithms include phosphoRS (Taus et al., 2011), Ascore (Beausoleil et al., 2006), Andromeda's PTM Score (Cox et al., 2011) and recently released PTMProphet (Shteynberg et al., 2019). We have previously benchmarked performance of some of the instruments and software pipelines for phosphoproteomics (Ferries et al., 2017), demonstrating that there is considerable variability in how such scores map to robust statistics, such as local or global FLR, depending on instrument fragmentation mode and resolution.

Following confident identification of phosphopeptides and localisation of given sites, data tend to be compiled from within a single study or across multiple studies (meta-analysis) to determine the extent of evidence for a given site from multiple PSMs. In general, where there are independent observations of PSMs supporting a phosphosite, it can be reasonably assumed that the evidence for a site to be real increases, although to our knowledge there are no current statistical models to calculate this phenomenon accurately. Multiple PSMs can be observed per identified phosphosite as a result of either different peptide sequences containing that site, or the same peptide sequence being detected several times (Dephoure et al., 2013). There are some caveats to this logic though, as it is possible for the same PSM to be wrongly assigned to a phosphopeptide multiple times. This can occur if the correct interpretation for the spectrum had a very similar peptide sequence and identical mass to the wrongly assigned phosphopeptide (Chalkley and Clauser, 2012; Lee, Jones and Hubbard, 2015). Although LC-MS/MS and computational analysis is generally recognised as very effective and reliable for phosphosite detection, from each study it is likely that there is some element of remaining false discovery of peptides and sites wrongly localised, depending on the statistical thresholds applied. This is particularly problematic for studies that set relatively weak thresholds (e.g., equating to site probability $>0.75$ ) in order to maximise sensitivity - more true positives may be gained, but at the expense of very large numbers of false positives passing the threshold. Methods and guidelines for FLR are still evolving and not consistently applied in published phosphoproteome studies, and so it is likely that most published studies contain considerable numbers of falsely localised phosphosites (Desiere et al., 2006; Dinkel et al., 2011; Gnad, Gunawardena and Mann, 2011; Hornbeck et al., 2015). This can lead to overestimation of the total number of known true human phosphosites if database providers do not control for FDR across multiple data sets (Ochoa et al., 2020). 
bioRxiv preprint doi: https://doi.org/10.1101/2021.04.14.439901; this version posted April 15, 2021. The copyright holder for this preprint (which was not certified by peer review) is the author/funder, who has granted bioRxiv a license to display the preprint in perpetuity. It is made available under aCC-BY 4.0 International license.

One such database is PhosphoSitePlus (PSP) which represents a comprehensive, manually-curated and wellcited resource containing experimentally defined PTMs primarily focusing on phosphorylation (Hornbeck et al., 2015). As of March 2020, PSP encompassed phosphosite identification evidence across 17830 human protein sequences. The evidence for phosphorylation comes from manually-curated reviews of literature describing tandem MS and low-throughput experiments (Hornbeck et al., 2015). Interestingly, the majority of phosphosites in PSP only have 1 piece of evidence associated with their identification, and, as mentioned in the PSP documentation itself, the researchers must be cautious when accepting such sites as true positives (Hornbeck et al., 2015). It is possible that many users of PSP are not aware of the need for caution when reviewing or re-using data downloaded in bulk, and we are not aware of any previous effort to assess phosphosite FDR within PSP. A second curated proteomics resource is PeptideAtlas (PA) (Desiere et al., 2006) which is a repository of tandem MS datasets that have been processed through Trans-Proteomic Pipeline to ensure high and consistent quality of phosphopeptide identifications (Deutsch et al., 2010). The latest PA builds incorporate the use of the PTMProphet algorithm for phosphosite localisation where each potential phosphosite within an observed PSM is assigned a probability score between 0 and 1 of being phosphorylated (Shteynberg et al., 2019). As with PSP datasets, researchers must be cautious when accepting sites in PA with only 1 PSM as positively identified phosphosites. Instead, phosphosites that not only have multiple PSM observations in PA, but also have high phosphorylation probability scores assigned within the majority of those PSMs are most likely to be true positive identifications.

The issue of FDR within human phosphorylation databases is undeniably important, although to our knowledge no estimates have been made to predict its scale across large datasets. In this work, by profiling the human phosphoproteome, we aimed to estimate the false discovery rate of phosphosites with evidence in PSP and/or PA and use these estimates to predict the count of true phosphosites within the proteome. We categorised the sites into sets of various predicted phosphorylation likelihood based on the amount of positive identification evidence they have in PSP and PA. By using orthogonal properties of phosphosites assigned to the sets, such as evolutionary conservation, sequence properties and functional annotations, we aimed to demonstrate significant differences between the sets and develop an improved method for independent FDR estimation which can be used to indicate the extent of true phosphosites within the human phosphoproteome. 
bioRxiv preprint doi: https://doi.org/10.1101/2021.04.14.439901; this version posted April 15, 2021. The copyright holder for this preprint (which was not certified by peer review) is the author/funder, who has granted bioRxiv a license to display the preprint in perpetuity. It is made available under aCC-BY 4.0 International license.

\section{Methods}

\section{Processing and categorising phosphorylation data in PSP and PA}

Phosphorylation data in PeptideAtlas (PA) (2020 build) (Desiere et al., 2006) was filtered to only include human Ser/Thr/Tyr sites from canonical UniProt protein sequences with at least 1 PSM observation $(1,069,709$ sites across 63,616 sequences) (SI Table 1). The sites were categorised according to the number of PSM observations with a certain phosphorylation probability score assigned by PTMProphet (Shteynberg et al., 2019). The counts of observations with a probability of $>0.95$ were used as "positive" evidence for site phosphorylation. The counts at a probability threshold of $\leq 0.19$ were used as "negative" evidence in favour of a site being a non-phosphosite. The total number of PSM observations per site was considered to distinguish sites for which $\geq 10 \%$ of all associated PSMs had a PTM probability $>0.95$, from sites where a small minority $(<10 \%)$ of associated PSMs had this probability. Based on this, selected confidence categories were applied to predict site phosphorylation likelihood in PA ("High": $\geq 5$ positive observations which accounted for $\geq 10 \%$ of total observations across all probabilities; "Medium": $\geq 5$ positive observations which accounted for $<10 \%$ of total observations or 2-4 positive observations; "Low": 1 positive observation; "Not phosphorylated": 0 positive observations and $\geq 5$ negative observations; "Other": site did not fall into any described categories). PhosphoSitePlus (PSP) data (11/03/20 build; Phosphorylation_site_dataset.gz) (Hornbeck et al., 2015) was filtered to only include human Ser/Thr/Tyr sites from canonical protein sequences labelled by UniProt identifiers (231,607 sites across 17,830 sequences) (SI Table 2). The sites were ranked based on the number of times they have been characterised in low/high throughput studies. The sum of observations across all studies was used to predict site phosphorylation likelihood in PSP ("High": $\geq 5$ observations; "Medium": 2-4 observations; "Low": 1 observation).

\section{$\underline{\text { Evolutionary conservation analysis }}$}

To determine the cross-species conservation of all Ser, Thr and Tyr sites in the reference human proteome (The UniProt, 2019) which have phosphorylation evidence in PSP and PA, human reference proteome $(20,605$ sequences, UniProt ID: UP000005640) and the proteomes of 100 eukaryotic species (50 mammals, 12 birds, 5 fish, 4 reptiles, 2 amphibians, 11 insects, 4 fungi, 7 plants and 5 protists; SI Table 3) were downloaded from UniProt (UniProt release 2019_10). Each sequence in the human proteome was used as a query in a BLASTp search (BLAST+ 2.10 .0 version) (Altschul et al., 1990) against all 100 eukaryotic proteomes. The BLAST output (SI Table 4) was processed to extract a top matching significant orthologue ( $E$-value of $\leq 0.00001$ ) from each species for each human target. Human targets were then aligned with their matched orthologues using the MUSCLE algorithm (version 3.8.31) (Edgar, 2004) with default settings if all sequences to be aligned were $<2,000$ amino acids long. If any sequences to be aligned (either the human sequence or any of the orthologue sequences) were $\geq 2,000$ amino acids long, 2 iterations of the algorithm were run using settings for large alignments (-maxiters 2 option) (Edgar, 2004). From the alignments, percentage conservation scores were calculated for every Ser, Thr and Tyr site within each human target out of 100 (all eukaryotic proteomes) and out of the number of aligned orthologues. Conservation percentages were calculated considering any Ser/Thr substitutions in orthologues, whereby an orthologue was included in the count if, for example, a Thr in its sequence was aligned with a Ser in the target human sequence and vice versa. Conservation data was then cross-referenced with PSP/PA datasets to identify sites in the human proteome with phosphorylation evidence in PSP/PA and determine their conservation. To ensure consistency in terms of proteins and sites used, any human protein target for which it was not possible to calculate site conservation either due to the protein having no matches in BLAST (14 proteins), no significant matches in BLAST (236 proteins), no Ser/Thr/Tyr sites in its sequence (1 protein) or due to failed alignments (10 proteins), was excluded from any further analysis (SI Table 5). Any human targets labelled with the same UniProt identifier in the reference human proteome, PSP and PA, but which corresponded to different protein sequences across the datasets ( 73 proteins; SI Table 6) were also excluded. Conservation was assessed for the remaining targets (SI Material 1) by linear regression models with non-assumed intercept for simpler interpretation of slope between phosphosites and nonphosphosites. Average conservation of likely phosphosites (sites ranked "High" or "Medium" in PSP and/or PA) was plotted against average conservation of likely non-phosphosites (sites in "Not phosphorylated" and 
bioRxiv preprint doi: https://doi.org/10.1101/2021.04.14.439901; this version posted April 15, 2021. The copyright holder for this preprint (which was not certified by peer review) is the author/funder, who has granted bioRxiv a license to display the preprint in perpetuity. It is made available under aCC-BY 4.0 International license.

"Other" sets) within each target protein that had at least 3 likely phosphosites and 3 likely non-phosphosites. Conservation scores (\%) were also compared across all sites within phosphorylation likelihood sets using box plots.

\section{Analysis of amino acids adjacent to phosphosites}

Target protein sequences (20,271 sequences; SI Material 2) were processed to identify amino acids at the -1 and +1 proximal positions adjacent to every Ser, Thr and Tyr site. If a target sequence ended with a Ser, Thr or Tyr site then its +1 amino acid was marked as "Not found". For each amino acid, its frequency at each proximal position was first normalised to 1,000 and then to its frequency in the pre-filtered human reference proteome (expected distribution). Proximal amino acid frequencies around target Ser, Thr and Tyr in "High in PSP and PA" set were compared to those in the "Not phosphorylated" set, and to the expected amino acid distribution. The comparisons were assessed by Fisher's exact statistical test (Fisher, 1934) performed using scipy module in Python (Virtanen et al., 2020) with Bonferroni corrections to generate adjusted p-values. For each amino acid, any significant difference (Bonferroni corrected p-value $<0.01$ ) between the compared sets was used to estimate phosphosite false discovery rate across all phosphorylation likelihood sets. FDR estimates assumed that all sites in the highest phosphorylation likelihood set "High in PSP and PA" set were true positive phosphosite identifications, whereas all sites with the weakest phosphorylation confidence (either the "Not phosphorylated" or the "Other" set) were non-phosphosites. Therefore, the observed count of a certain proximal amino acid in the "High in PSP and PA" (nPos) corresponded to its expected count at $0 \%$ FDR, whereas its observed count in the "Not phosphorylated" or "Other" set (nNeg) corresponded to its expected count at $100 \%$ FDR. To estimate \% FDR in any other phosphorylation likelihood set based on the observed count of the compared proximal amino acid in that set (nObs), we used the following equation:

$$
\% \mathrm{FDR}=\left(1-\frac{\mathrm{nObs}-\mathrm{nNeg}}{\mathrm{nPos}-\mathrm{nNeg}}\right) \times 100
$$

The equation has the effect of estimating what proportion of the observed count (nObs) is explained by assumed false positives (nNeg) and what proportion by true positives (nPos). For example, if amino acid X was found at +1 position next to 500 Ser sites in the highest phosphorylation confidence set $(0 \%$ FDR set; nPos $=500)$ compared to 10 Ser sites in the "Not phosphorylated" set $(100 \%$ FDR set; nNeg $=10)$, and next to 350 sites in the set of interest (nObs $=350$ ), then pSer FDR within the set of interest would be $30.6 \%$. This would suggest that $30.6 \%$ of sites in that set behave like false positive pSer in terms of $\mathrm{X}$ amino acid frequency at +1 position, whereas $69.4 \%$ of those sites behave like sites in the highest phosphorylation likelihood set (true pSer).

An average FDR with 95\% confidence intervals (CI) was calculated per each likelihood set if multiple amino acids were significantly enriched at adjacent positions around a particular target phosphosite. Final FDR estimates were used to derive the total number of true positive (TP) phosphosite identifications across phosphorylation likelihood sets.

To compare FDR/TP estimates between individual PSP and PA sets, the method was replicated using alternative phosphorylation likelihood sets, where sites were categorised according to the highest amount of positive phosphorylation evidence from one database (at least 1 observation at PTM probability $>0.95$ in PA; at least 1 observation in PSP), without taking into account any evidence in the other. Phosphosite FDR estimates within "High" sets in each database were presented as a weighted average between FDR estimates in sites ranked "High" in that database only and sites ranked "High" in both PSP and PA. For example, the FDR in "High in PA" set was a weighted average of FDR estimates in "High in both" set and "High in PA only" set.

\section{Functional enrichment analysis}

All protein sequences in the filtered reference human proteome (SI Material 2) were categorised into sets according to what their highest ranked Ser, Thr and Tyr site was in terms of phosphorylation evidence ("High in PSP and PA", "High in PSP or PA", "Medium in PSP and/or PA", "Low in PSP and/or PA", "Other in PA", "Not phosphorylated" and "No evidence in PSP or PA"). Each protein set within Ser, Thr and Tyr datasets (SI 
bioRxiv preprint doi: https://doi.org/10.1101/2021.04.14.439901; this version posted April 15, 2021. The copyright holder for this preprint (which was not certified by peer review) is the author/funder, who has granted bioRxiv a license to display the preprint in perpetuity. It is made available under aCC-BY 4.0 International license.

Material 1) was analysed with DAVID (version 6.8) (Dennis et al., 2003) using all proteins in filtered proteome with any Ser, Thr or Tyr evidence in PSP or PA (16,296, 14,565 and 12,912 proteins respectively) as control background. Protein sets containing no reported evidence in PSP or PA were searched against a background of all proteins in the filtered reference proteome to determine any differences in their functional enrichment compared to proteins with PSP/PA evidence. Per each set searched, the top 10 (where possible) significant (Benjamini-Hochberg corrected $\mathrm{p}$ value $<0.05$ ) functional terms with the highest percentage of proteins mapped were identified, replacing any near synonymous terms with additional terms from outside the initial top 10. All target protein sets were also searched in UniProt (release 2020_04) to determine percentage of proteins mapped to UniProt keywords "Phosphoprotein", "Alternative splicing", "Nucleus", "Transcription", "Acetylation", "Membrane", "Glycoprotein", "Signal" and "Disulfide bond".

\section{$\underline{\text { Secondary structure analysis }}$}

Categorised Ser, Thr and Tyr sites in filtered reference human proteome were mapped to protein structures (beta strand, helix, turn and coiled coil) described for those proteins in UniProt (release 2020_04) (SI Material 1, SI Table 7). Any target proteins searched in UniProt which were marked as obsolete (15 proteins) or represented different sequences despite being labelled with the same identifier ( 25 proteins) were removed from the analysis and marked as "NA" (SI material 1). Normalised (to 1,000) counts of target amino acids within protein structures were assessed with Fisher's exact statistical test (Fisher, 1934) using the scipy module in Python (Virtanen et al., 2020) to generate p-values and indicate any significant enrichment $(\mathrm{p}<0.05)$ between "High in PSP and PA" set and the "Not phosphorylated" set. 


\section{Results and discussion}

$\underline{\text { Categorising all Ser, Thr and Tyr annotated phosphosites in the human proteome }}$

We first ranked all Ser, Thr and Tyr phosphosites in PA and PSP in the filtered reference human proteome according to the amount of accumulated identification evidence (Fig. 1; Table 1; SI material 1). We found that the majority of Ser, Thr and Tyr sites $(50.1 \%, 63.3 \%$ and $54.3 \%$ respectively) with phosphorylation evidence in PSP were placed into the "Low" phosphorylation likelihood set meaning that there was only 1 piece of evidence describing their positive identification (Fig. 1a). Furthermore, out of all analysed Ser, Thr and Tyr sites with at least 1 observation at PTM probability $>0.95$ in PA (suggesting a positive phosphosite identification), $21.7 \%, 34.0 \%$ and 33.5\% respectively were placed in the "Low" set (Fig. 1b, Table 1), highlighting that a considerable amount of potential phosphosites only had 1 piece of positive identification evidence across both databases.

Interestingly, we found that in the human proteome there were more Tyr sites assigned to "High" set in PSP (5+ observations) than Thr sites (Fig. 1a, Table 1). Assuming that sites in the "High" set are likely to be real phosphosites, this finding contradicts the widely accepted notion that Thr phosphosites are more abundant than Tyr phosphosites (Hardman et al., 2019; Olsen et al., 2006), although this may vary depending on the active cell cycle phase or exposure to growth-factors (Caron et al., 2016). High prevalence of likely true Tyr phosphosites in the PSP dataset could have been a result of in-house studies which identified large numbers of pTyr sites using immunoaffinity strategies not suitable for pSer/pThr discovery (Rikova et al., 2007; Rush et al., 2005), and studies which have not been officially published (Hornbeck et al., 2015).

A

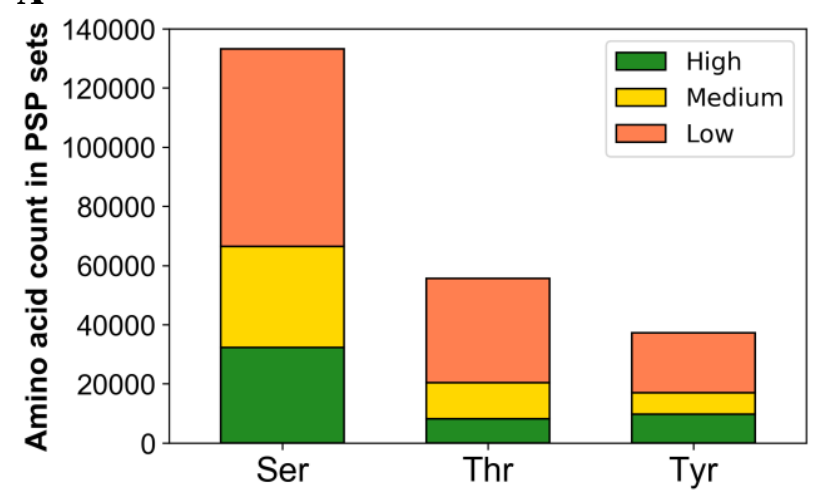

C

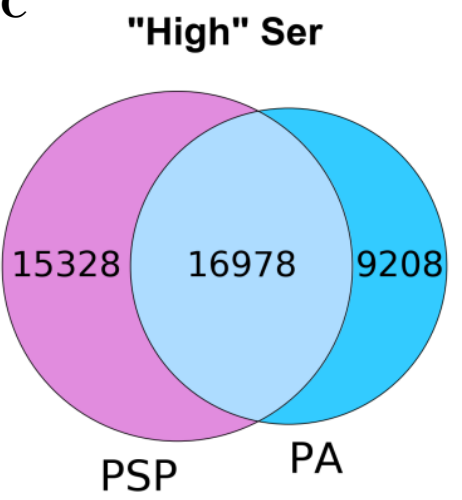

B

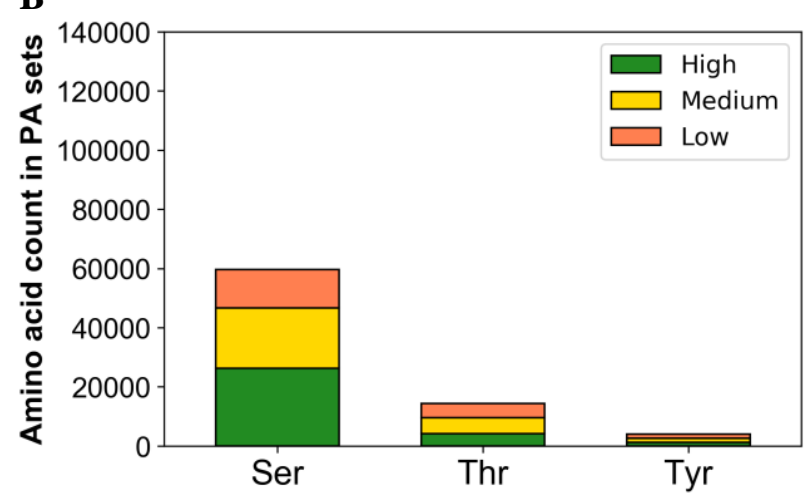

D

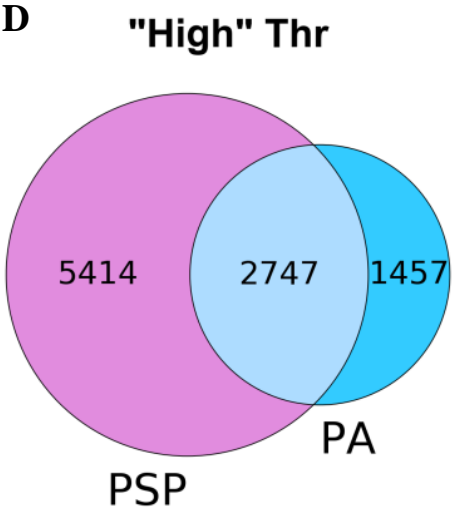

$\mathbf{E}$

E "High" Tyr

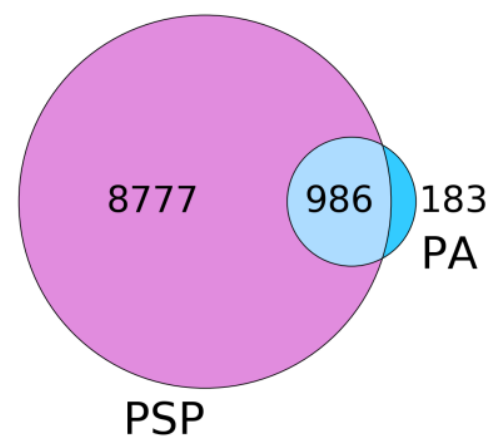

Figure 1. Distribution of serine (Ser), threonine (Thr) and tyrosine (Tyr) phosphosites from UniProt's reference human proteome that have any positive identification evidence in (A) PhosphoSitePlus (PSP) or (B) PeptideAtlas (PA) based on established phosphorylation likelihood sets (see "Methods"). Venn diagrams provide the counts of (C) Ser, (D) Thr and (E) Tyr sites ranked "High" in PSP (left), PA (right) and both resources (overlap). 
bioRxiv preprint doi: https://doi.org/10.1101/2021.04.14.439901; this version posted April 15,2021 . The copyright holder for this preprint (which was not certified by peer review) is the author/funder, who has granted bioRxiv a license to display the preprint in perpetuity. It is made available under aCC-BY 4.0 International license.

From PA, it is possible to identify sites for which covering phosphopeptides are observed but for which the modifications are only localised to other sites in the same peptides, thus providing strong evidence for likely non-phosphosites. Sets of potential Ser, Thr and Tyr non-phosphosites were therefore established based initially on evidence in PA (SI Table 8). Those sets were then cross-referenced with data in PSP to determine whether PSP contained any sites ranked as non-phosphosites in PA. Interestingly, we found that 2,489 Ser, 1,341 Thr and 891 Tyr sites assigned to the "Not phosphorylated" set in PA were found to have evidence in PSP (SI Table 9). In fact, out of those potential PA non-phosphosites, $146 \mathrm{Ser}, 97 \mathrm{Thr}$ and $293 \mathrm{Tyr}$ sites were placed into "High" phosphorylation likelihood set according to PSP evidence (SI Table 9). This strongly indicated the presence of potential false positives in PSP and/or false negatives in PA. For example, sites Ser42 in protein P17066 (HSPA6) and Ser59 in Q8N488 (RYBP) had 8 and 6 phosphosite identification references in PSP respectively (mostly from in-house MS studies) but had no positive identification evidence in PA or any mention in other databases including UniProt (The UniProt, 2019) and neXtProt (Zahn-Zabal et al., 2020) (SI material 1). On the other hand, site Ser4 in P15927 (RPA2) had 33 phosphosite identification references in PSP and was also mentioned in UniProt's and neXtProt's annotations, but has never been positively localised in any of its 127 associated PSMs in PA (SI material 1). To eliminate potential false assignments when considering evidence in both PSP and PA, a site was only categorised as a non-phosphosite if it had no evidence in PSP in addition to having "negative" phosphorylation evidence in PA (Table 1). As a result, we established final negative control sets containing 13,892 Ser, 8,462 Thr and 2,184 Tyr sites. Similar adjustments were made to the "Other" PA set (sites in that set must have no evidence in PSP) which contained the majority of analysed PA sites (Table 1).

Table 1. Categorising serine (Ser), threonine (Thr) and tyrosine (Tyr) sites from UniProt's reference human proteome into phosphorylation likelihood sets based on available phosphorylation evidence in PhosphoSitePlus (PSP) and PeptideAtlas (PA).

\begin{tabular}{|c|c|c|c|c|}
\hline Phosphorylation likelihood set & Phosphorylation evidence per site & Ser count & Thr count & Tyr count \\
\hline High in PSP & $5+$ pieces of evidence & 32306 & 8161 & 9763 \\
\hline Medium in PSP & 2-4 pieces of evidence & 34154 & 12197 & 7228 \\
\hline Low in PSP & 1 piece of evidence & 66777 & 35173 & 20191 \\
\hline High in PA & $\begin{array}{l}5+\text { observations at PTM score }>0.95 \text { which } \\
\text { is } \geq 10 \% \text { of total observations }\end{array}$ & 26186 & 4204 & 1169 \\
\hline Medium in PA & $\begin{array}{l}5+\text { observations at PTM score }>0.95 \text { which } \\
\text { is }<10 \% \text { of total observations OR } 2-4 \\
\text { observations at PTM score }>0.95\end{array}$ & 20517 & 5297 & 1460 \\
\hline Low in PA & 1 observation at PTM score $>0.95$ & 12950 & 4895 & 1324 \\
\hline Not phosphorylated & $\begin{array}{l}0 \text { observations at } \mathrm{PTM} \text { score }>0.19 \text { AND } \\
5+\text { observations at PTM score } \leq 0.19 \text { AND } \\
\text { no evidence in PSP }\end{array}$ & 13892 & 8462 & 2184 \\
\hline Other sites & $\begin{array}{l}\text { At least } 1 \text { observation in PA but does not } \\
\text { fall into any other PA categories AND no } \\
\text { evidence in PSP }\end{array}$ & 60221 & 35000 & 10009 \\
\hline
\end{tabular}

Having further cross-referenced sets of sites of various phosphorylation likelihood between PSP and PA (SI Table 9), we established a "gold standard" set of phosphosites, all of which had "High" phosphorylation likelihood according to both PSP and PA evidence. This set contained 16,978 Ser (Fig. 1c), 2,747 Thr (Fig. 1d) and 986 Tyr (Fig. 1e) highly likely true phosphosites. As for the general agreement between PSP and PA in terms of phosphorylation evidence, we found that $37.7 \%$ of Ser, $20.5 \%$ of Thr and $9.10 \%$ of Tyr sites with PSP evidence also had at least 1 observation at PTM probability $>0.95$ in PA (SI Table 9). This variation in sites observed between the two databases can be explained by the likely use of different methods for phosphosite detection and localisation between PA and the sources referenced in PSP, as well as due to a considerable presence of random false positives in both datasets before thresholding has been applied (see 'Introduction'). 


\section{$\underline{\text { Evolutionary conservation analysis }}$}

Phosphoproteomes from all species are constantly evolving, although many ancient phosphosites are conserved across species and taxa, where they are likely to be functionally relevant (Boekhorst et al., 2008; Malik, Nigg and Körner, 2008; Studer et al., 2016). In our analysis we determined the conservation of all potential Ser, Thr and Tyr phosphosites and non-phosphosites in UniProt's filtered reference human proteome across 100 eukaryotic species, weighed towards vertebrates, but also including examples of insects, plants and unicellular eukaryotes (SI Table 3). In our first analysis, we explored the mean conservation of phosphosites and non-phosphosites per protein (at least three of each per protein) and performed a correlation analysis across all proteins (Fig. 2). We fitted linear regression models through the origin, under the theory that proteins unique to humans would have zero conservation for both phosphosites and non-phosphosites. We found great variation between the conservation of both site types, ranging from near zero to $100 \%$, which was mostly dependent on the overall conservation of the protein sequence. However, based on the generated linear regression models, we concluded that on average, Ser, Thr and Tyr phosphosites ("High" or "Medium" in PSP and/or PA) were around $4.6 \%, 5.4 \%$, and $2.0 \%$ respectively more conserved across all 100 eukaryotes than corresponding likely non-phosphosites (sites in "Not phosphorylated" and "Other" sets) within analysed proteins when allowing Ser/Thr substitutions towards the conservation score (Fig. 2). Similar results were obtained when assessing phosphosite conservation only across found orthologues for each protein (SI Fig. 1). The results (Fig. 2, SI Fig. 1) provide additional evidence that phosphosites are generally more conserved that non-phosphosites (Boekhorst et al., 2008; Chen, Chen and Li, 2010; Malik, Nigg and Körner, 2008). The difference in conservation is thus subtle and variable, but statistically robust. Furthermore, in our analysed sets of proteins which had at least 3 likely phosphosites and 3 likely non-phosphosites, we found 104, 88 and 19 proteins where the conservation of Ser, Thr and Tyr likely phosphosites respectively was at least $20 \%$ higher than conservation of likely non-phosphosites (SI Table 10).
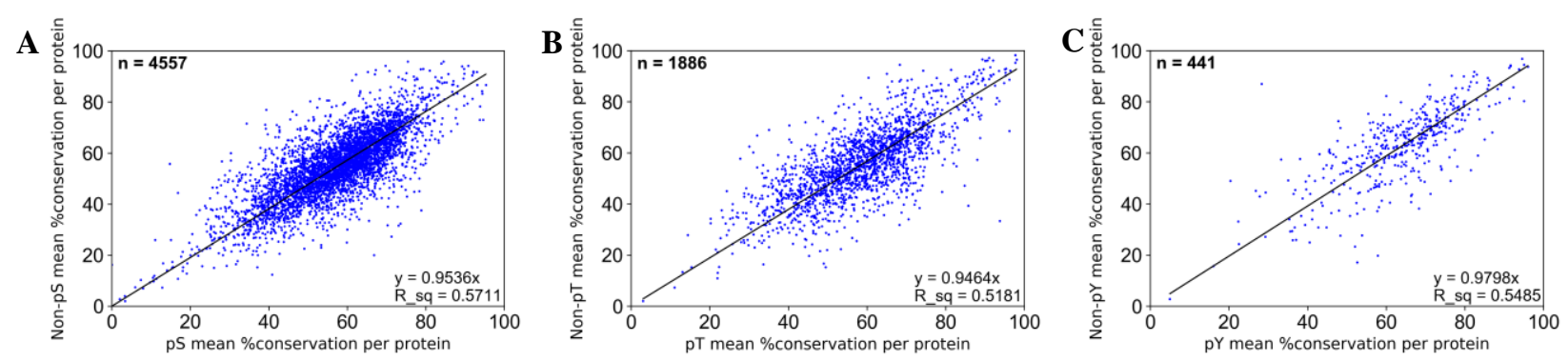

Figure 2. Mean \% conservation across 100 eukaryotic species of likely (A) Ser, (B) Thr, (C) Tyr phosphosites and corresponding likely non-phosphosites within each target protein $\left(n=\right.$ number of proteins analysed). The regression coefficient $\left(R^{2}\right)$ is given by ' $R \_s q$ '.

In our next analysis, we compared the conservation of all sites split by phosphorylation likelihood sets (Fig. 3) and revealed that sites in the highest phosphorylation likelihood set ("High in both PSP and PA") had the highest average conservation across all 100 eukaryotic proteomes considering Ser/Thr substitutions (average conservation of $58.4 \%, 58.6 \%$ and $69.4 \%$ across 16,978 Ser, 2,747 Thr and 986 Tyr sites respectively) (Fig. 3, SI Table 11). In comparison, the sites in "Low in PSP and/or PA" set had slightly lower average conservation scores of 54.3\%, 55.4\% and 64.0\% in 35126 Ser, $13253 \mathrm{Thr}$ and $7471 \mathrm{Tyr}$ sites respectively (Fig. 3, SI Table 11). Assuming that high conservation is a property of true phosphosites, the results (Fig. 3) show that this property was observed more frequently in higher phosphorylation likelihood sets compared to lower ones suggesting higher potential phosphosite FDR in sets with weaker phosphorylation evidence. 


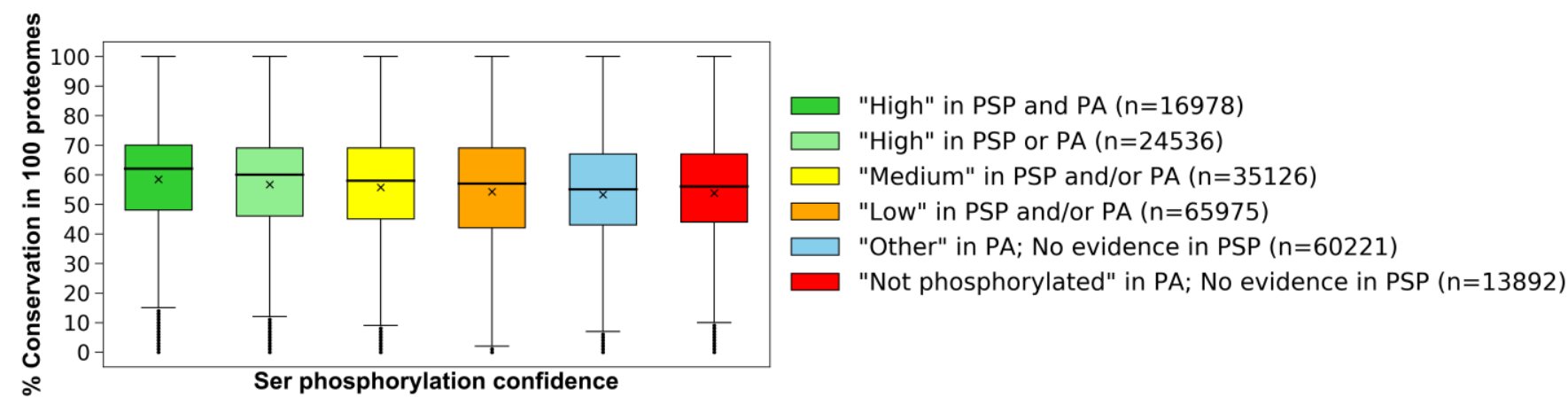

B

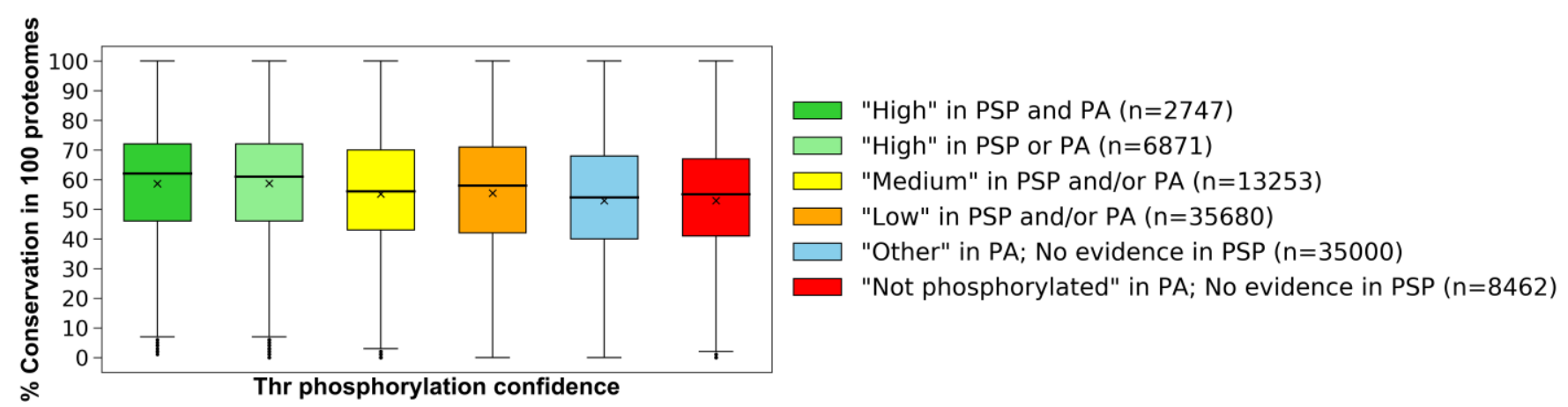

C

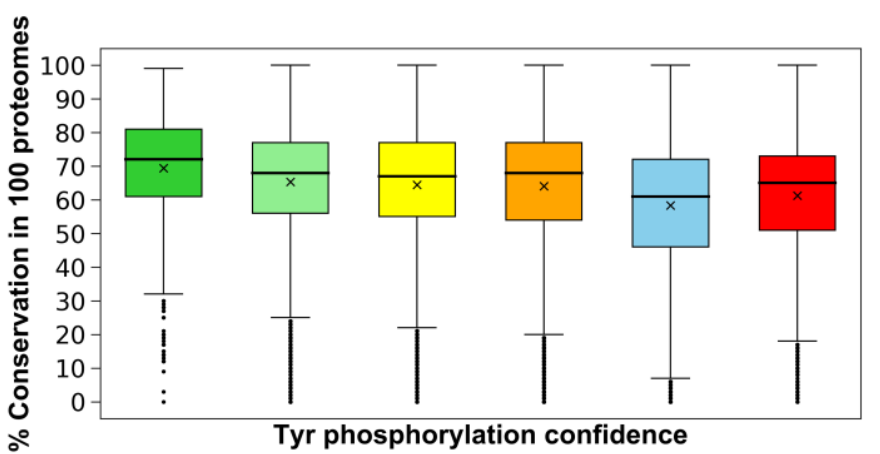

$\square$ "High" in PSP and PA $(n=986)$

"High" in PSP or PA $(n=8960)$

"Medium" in PSP and/or PA $(n=7471)$

"Low" in PSP and/or PA $(n=20333)$

"Other" in PA; No evidence in PSP ( $n=10009)$

"Not phosphorylated" in PA; No evidence in PSP $(n=2184)$

Figure 3. Box plots of conservation percentages (\%) across 100 eukaryotic species of human (A) Ser, (B) Thr and (C) Tyr sites categorised across established phosphorylation confidence sets based on PSP and PA evidence. Within each box, a horizontal line represents median \% conservation, an (x) symbol represents mean \% conservation per group. Each box extends from the 25 th to the 75th percentile of each set's distribution of conservation \% values. Vertical lines extending from the boxes correspond to adjacent values. Dots $(\bullet)$ represent outlier values.

There were numerous cases in our analysis of likely non-phosphosites and sites with "Low" phosphorylation likelihood where amino acid conservation was also high compared to likely phosphosites (SI material 1), indicative of a conserved function for these amino acids in, for example, catalysis or a biomolecular interaction that is unrelated to phosphorylation. Furthermore, we found 64, 30 and 6 proteins in which the average conservation across 100 eukaryotes of Ser, Thr and Tyr likely non-phosphosites respectively was at least $20 \%$ higher than the conservation of corresponding likely phosphosites (SI Table 10). It is possible that the predicted phosphosites within those proteins were either false positives or were non-functional true phosphosites, explaining the comparative weaker selective pressure. In fact, previous reports estimated that as many as $65 \%$ of known phosphosites may be non-functional due to limited kinase specificity and therefore have similar evolution rates compared to non-phosphosites which would explain the trends in our analysis (Landry, Levy and Michnick, 2009; Lienhard, 2008). It is also possible that some proteins could have been formed by recent 
bioRxiv preprint doi: https://doi.org/10.1101/2021.04.14.439901; this version posted April 15, 2021. The copyright holder for this preprint (which was not certified by peer review) is the author/funder, who has granted bioRxiv a license to display the preprint in perpetuity. It is made available under aCC-BY 4.0 International license.

gene fusion events leading to regions containing phosphorylation sites only found in a few closer related orthologues (low conservation), with other protein domains being more highly conserved.

\section{Analysis of amino acids adjacent to phosphosites}

Amino acids directly adjacent to known phosphorylation sites are often involved in optimising substrate capture for subsequent phosphotransfer by the kinase enzymatic machinery (Byrne et al., 2020; Hutti et al., 2004; Kettenbach et al., 2012). In our analysis we identified the frequency of -1 and +1 amino acids relative to a possible phosphosite and compared it across different sets of sites ranked by the relative strength of phosphorylation evidence (Table 1). We found a strong enrichment of proline (Pro) at the +1 position next to Ser and Thr sites in the reference human proteome that were placed in the set with the most phosphorylation evidence ("High" in PSP and PA") (Fig. 4a \& 4b, SI Table 12). In fact, Pro was observed at the +1 position next to $44.3 \%$ and $74.9 \%$ of all Ser and Thr sites respectively in that set (SI Table 12). The enrichment of Pro at +1 position around those sites was significant (adj. $p$ value $<0.01$ ) in relation to the normalised distribution of Pro in the human proteome, where it is, in fact, only the sixth most observed amino acid (SI Table 12). The normalised number of +1 observations of Pro around Ser and Thr sites in the highest phosphorylation likelihood set was also significantly (adj. $\mathrm{p}$ value $<0.01$ ) higher than around Ser and Thr sites in the "Not phosphorylated" set (Fig. 4a \& 4b), where only $2.68 \%$ of Ser and $5.67 \%$ of Thr sites had Pro at +1 position (SI Table 12). Therefore, the enrichment of Pro around highly likely Ser and Thr phosphosites suggests that this feature, amongst others, can be used as a differentiating characteristic for phosphosites compared to nonphosphosites. Furthermore, multiple previous reports specifically highlight the importance of Pro in the mechanism of phosphorylation for families of kinases such as the cyclin-dependent kinases, Mitogen Activated Protein Kinases and, more recently, the centrosomal kinase PLK4 (Byrne et al., 2020; Hall and Vulliet, 1991; Johnson et al., 1998; Keshwani et al., 2015; Lu, Liou and Zhou, 2002; Pietrangelo and Ridgway, 2019; Songyang et al., 1994). Consequently, there is a high prevalence of Pro in numerous phosphorylation motif sequences as part of Ser/Thr-Pro combinations (Amanchy et al., 2007; Sugiyama, Imamura and Ishihama, 2019). As a result, we compared the frequency of Pro across the sites within different phosphorylation likelihood sets and used the comparison to estimate phosphosite false discovery rate across those sets. We found that in both Ser and Thr datasets, the number of Pro observations at +1 position next to target sites decreased with the amount of phosphorylation evidence, suggesting higher phosphosite FDR in Ser/Thr sets with weaker phosphorylation evidence (Fig. $4 \mathrm{a} \& 4 \mathrm{~b}$ ). Using the counts of Pro at +1 position around Ser sites of various phosphorylation likelihood (SI Table 12) and working under the assumption of FDR $=0 \%$ in set 1 "High" in PSP and PA", we estimated Ser phosphosite FDR = 43.0\% in set 2 "High" in PSP or PA"; FDR = $67.5 \%$ in set 3 "Medium in PSP and/or PA"; FDR $=86.1 \%$ in set 4 "Low in PSP and/or PA" and FDR $=92.1 \%$ in the "Other" set. Using the same method for the Thr data, we estimated Thr phosphosite FDR $=55.1 \%$ in set 2; FDR $=81.8 \%$ in set 3; FDR $=95.4 \%$ in set 4 and FDR $=98.8 \%$ in the "Other" set. Our FDR estimates clearly highlight that the majority of Ser and Thr sites with just 1 piece of phosphosite identification evidence are likely false positive identifications and users of these databases can reasonably assume that if a site does not have multiple levels of evidence, then it is unlikely to represent a true phosphorylation site. 
bioRxiv preprint doi: https://doi.org/10.1101/2021.04.14.439901; this version posted April 15, 2021. The copyright holder for this preprint (which was not certified by peer review) is the author/funder, who has granted bioRxiv a license to display the preprint in perpetuity. It is made available under aCC-BY 4.0 International license.

A

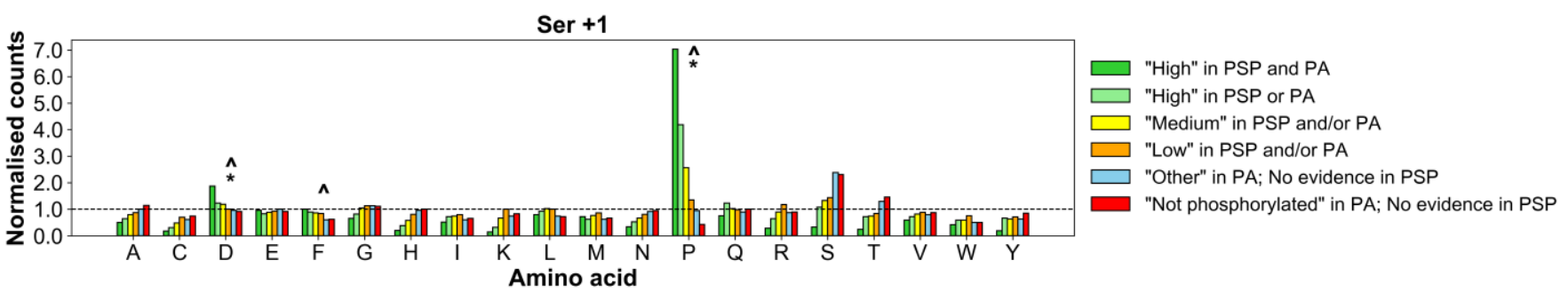

$\mathbf{B}$
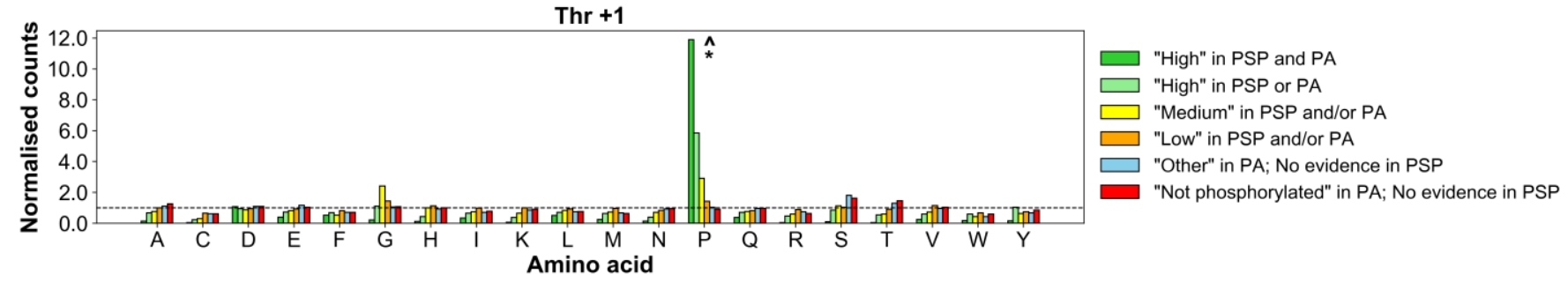

Tyr +1

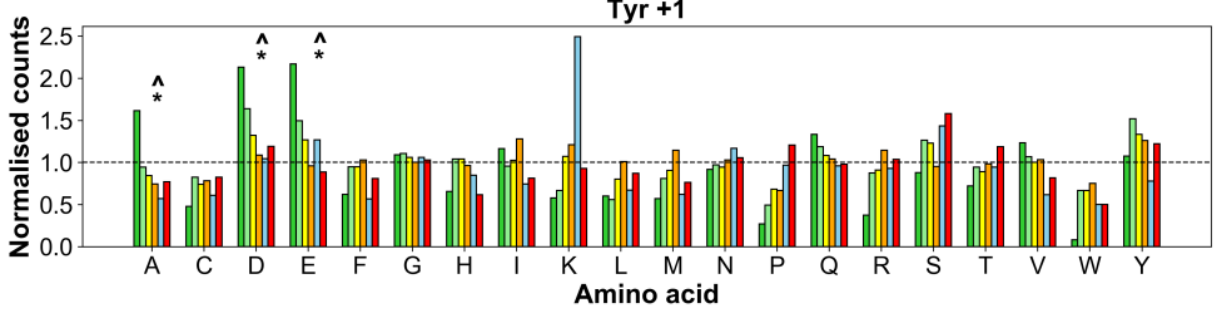

"High" in PSP and PA

$\square$ "High" in PSP or PA

"Medium" in PSP and/or PA

$\square$ "Low" in PSP and/or PA

$\square$ "Other" in PA; No evidence in PSP

"Not phosphorylated" in PA; No evidence in PSP

$\mathbf{D}$
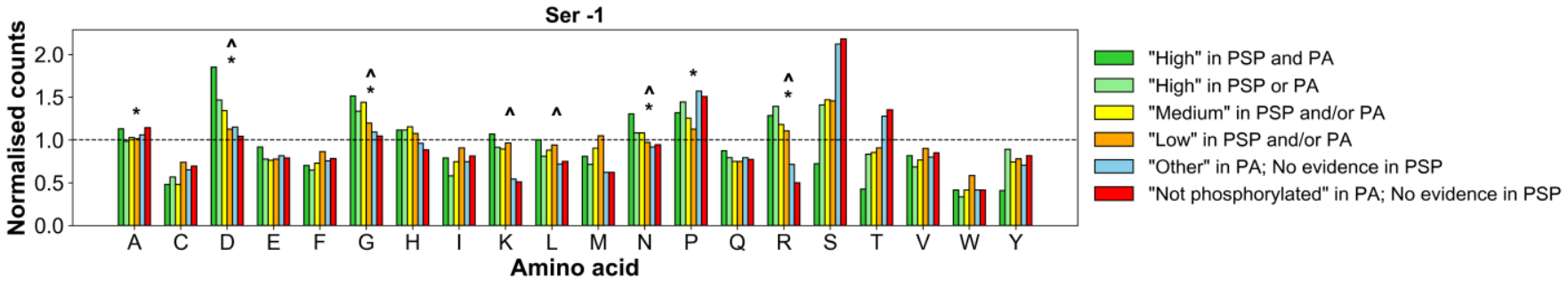

$\mathbf{E}$
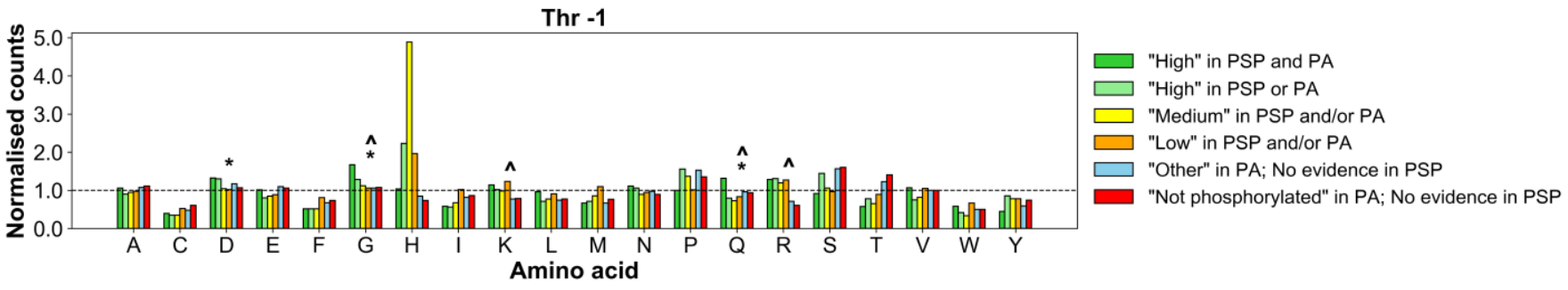

$\mathbf{F}$

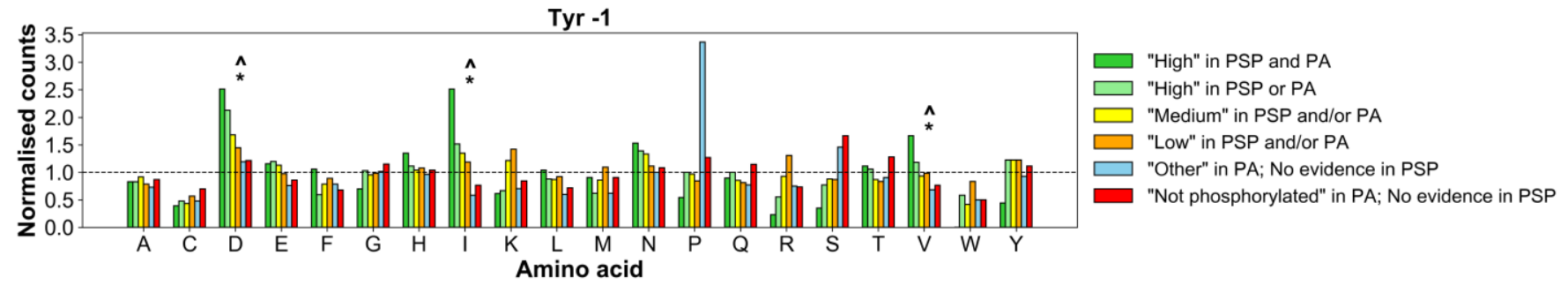

Figure 4. Counts of proximal amino acids positioned at (A) +1 around Ser; $(\mathbf{B})+1$ around Thr; (C) +1 around Tyr; (D) -1 around Ser; (E) -1 around Thr; (F) -1 around Tyr sites of various phosphorylation likelihood based on evidence in PSP and PA, normalised to observed distribution of those amino acids in human proteome (represented by dotted baseline set at 1). Significant (Bonferroni corrected $\mathrm{p}$ value $<0.01$ ) enrichment of proximal amino acids in the "High" in PSP and PA" set is highlighted by the caret symbol (^) when compared against the "Not phosphorylated" set, and an asterisk symbol (*) when compared to the expected amino acid distribution. 
We also found a significant enrichment of aspartic acid (Asp or D) at +1 position next to Ser sites in the highest phosphorylation likelihood set (Fig. 4a). To explain this, we linked the sequences containing those sites to Casein kinase II phosphorylation motifs which commonly feature Ser-Asp combinations (Amanchy et al., 2007; Songyang et al., 1996). At the -1 positions around target Ser, we found significant enrichment (adj. p value <0.01) of Asp, glycine (Gly or G), asparagine (Asn or $\mathrm{N}$ ) and arginine (Arg or R) in the highest phosphorylation likelihood set compared to "Not phosphorylated" set (Fig. 4d). It is possible that the observed enrichment was due to the presence of those amino acids within substrate motifs of Casein Kinase II, CDK5, PKC, PKA and MEKK (Amanchy et al., 2007), suggesting high prevalence of potential true Ser phosphosites. Similar conclusions were made for the enrichment of Gly at -1 around Thr sites in the highest phosphorylation likelihood set (Fig. 4e) which was linked to possible Gly-Thr combinations within PKA, ERK1 and ERK2 kinase substrate motifs (Amanchy et al., 2007). The enrichment of highlighted amino acids at the -1 position around Ser/Thr sites in the highest phosphorylation likelihood set (Fig. $4 \mathrm{~d} \& 4 \mathrm{e}$ ) was not as strong as the enrichment of Pro at +1 position (Fig. $4 \mathrm{a} \& 4 \mathrm{~b}$ ), and not as well cited as Pro in relation to phosphorylation (Hall and Vulliet, 1991; Keshwani et al., 2015; Lu, Liou and Zhou, 2002; Pietrangelo and Ridgway, 2019). As a result, we only relied on Pro enrichment at +1 positions to maximise the accuracy of FDR estimations within the sets of Ser and Thr sites.

In our analysis of proximal sites around target Tyr, we found a significant enrichment (adj. p value <0.01) of alanine (Ala or A), glutamic acid (Glu or E) and Asp at +1 positions, in addition to enriched isoleucine (Ile or I), valine (Val or V) and Asp at -1 positions in "High in PSP and PA" set compared to "Not phosphorylated" set (Fig. 4c \& 4f). We were able to link the enrichment of those proximal sites to their possible involvement in various phosphorylation motifs including EGFR and Abl kinase substrate motifs; PTP1B and PTPRJ phosphatase substrate motifs, and multiple SH2 domain binding motifs (Amanchy et al., 2007; Wälchli et al., 2004), therefore indicating higher frequency of true Tyr phosphosites in the highest confidence set compared to other sets. Since there was not a single proximal amino acid strongly enriched as, for example, Pro at +1 in Ser/Thr analysis, we used the frequencies of all six enriched proximal amino acids around target Tyr in "High in PSP and PA" (Fig. 4c \& 4f, SI Table 12) to estimate average Tyr phosphosite FDR with 95\% confidence intervals. We estimated FDR $=48.7 \%(\mathrm{CI} \pm 9.29 \%)$ in set 2 "High in PSP or PA", FDR $=69.4 \%(\mathrm{CI} \pm 5.08 \%)$ in set 3 "Medium in PSP and/or PA", FDR $=82.2 \%(\mathrm{CI} \pm 9.42 \%)$ in set 4 "Low in PSP and/or PA", and FDR $=97.8 \%(\mathrm{CI} \pm 4.38 \%)$ in the "Other" set (Table 2a; SI Table 13).

Based on our Ser, Thr and Tyr phosphosite FDR estimates, we predicted that there were 56,308 Ser, 10,305 Thr and 11,717 Tyr true positive (TP) phosphosite identifications in the human proteome that were supported by evidence in PSP and/or PA (Table 2a). Furthermore, the results suggested that 91,064 Ser, 48,666 Thr and 26,257 Tyr sites with positive phosphorylation evidence in PSP and/or PA (sites in "High", "Medium", "Low" sets) were false positives (Table 2a). Interestingly, the estimated count of Tyr TPs was higher than the count of Thr TPs which goes against the general understanding of threonine phosphorylation being more prevalent than tyrosine (Olsen et al., 2006). One explanation for this could be that initially there were more Tyr sites with strong phosphorylation evidence than Thr sites, particularly in PSP (Fig. 1a, Table 2a). Furthermore, it is possible that some sites in the "Not phosphorylated" and "Other" sets were false negatives. In fact, we found 6,626 Thr sites which had no positive phosphorylation evidence in PSP or PA but were mentioned as phosphosites in UniProt and/or neXtProt (SI material 1), suggesting possible underestimation of true positive Thr phosphosites in our analysis. 
bioRxiv preprint doi: https://doi.org/10.1101/2021.04.14.439901; this version posted April 15, 2021. The copyright holder for this preprint (which was not certified by peer review) is the author/funder, who has granted bioRxiv a license to display the preprint in perpetuity. It is made available under aCC-BY 4.0 International license.

Table 2. Counts of estimated true positive (TP) serine (Ser), threonine (Thr) and tyrosine (Tyr) phosphosites within sets of various phosphorylation likelihood based on (A) overall combined evidence and (B) individual positive identification evidence in PhosphoSitePlus (PSP) or PeptideAtlas (PA). Per each set, TP counts were derived from the FDR estimates within the set and the overall count of target amino acids in the set.

\begin{tabular}{|c|c|c|c|c|c|c|c|c|c|}
\hline Phosphorylation likelihood & $\begin{array}{c}\text { Ser } \\
\text { count }\end{array}$ & Ser FDR (\%) & $\begin{array}{l}\text { Ser TP } \\
\text { count }\end{array}$ & $\begin{array}{c}\text { Thr } \\
\text { count }\end{array}$ & Thr FDR (\%) & $\begin{array}{l}\text { Thr TP } \\
\text { count }\end{array}$ & $\begin{array}{c}\text { Tyr } \\
\text { count }\end{array}$ & $\begin{array}{l}\text { Tyr FDR (\%) } \\
\text { (CI) }\end{array}$ & $\begin{array}{l}\text { Tyr TP } \\
\text { count }\end{array}$ \\
\hline High in PSP or PA & 24536 & 43.0 & 13986 & 6871 & 55.1 & 3085 & 8960 & $48.7( \pm 9.29)$ & 4596 \\
\hline Low in PSP and/or PA & 65975 & 86.1 & 9171 & 35680 & 95.4 & 1641 & 20333 & $82.2( \pm 9.42)$ & 3626 \\
\hline Other in PA; No evidence in PSP & 60221 & 92.1 & 4757 & 35000 & 98.8 & 420 & 10009 & $97.8( \pm 4.38)$ & 224 \\
\hline $\begin{array}{l}\text { Not phosphorylated in PA; No } \\
\text { evidence in PSP }\end{array}$ & 13892 & 100 & 0 & 8462 & 100 & 0 & 2184 & 100 & 0 \\
\hline Phosphorylation likelihood & $\begin{array}{l}\text { Ser } \\
\text { count }\end{array}$ & Ser FDR (\%) & $\begin{array}{l}\text { Ser TP } \\
\text { count }\end{array}$ & $\begin{array}{l}\text { Thr } \\
\text { count }\end{array}$ & Thr FDR (\%) & $\begin{array}{l}\text { Thr TP } \\
\text { count }\end{array}$ & $\begin{array}{c}\text { Tyr } \\
\text { count }\end{array}$ & $\begin{array}{l}\text { Tyr FDR (\%) } \\
\text { (CI) }\end{array}$ & $\begin{array}{l}\text { Tyr TP } \\
\text { count }\end{array}$ \\
\hline High in PA & 26186 & 7.45 & 24235 & 4204 & 7.28 & 3898 & 1169 & $5.20( \pm 18.0)$ & 1108 \\
\hline High in PSP & 32306 & 26.7 & 23680 & 8161 & 42.6 & 4684 & 9763 & $44.6( \pm 10.8)$ & 5409 \\
\hline Total in PA & 59653 & & 39868 & 14396 & & 7231 & 3953 & & 2557 \\
\hline Total in PSP & 133237 & & 47447 & 55531 & & 9229 & 37182 & & 12053 \\
\hline
\end{tabular}

Using the same method, the analysis of Pro frequency at +1 position adjacent to target Ser/Thr sites, and the frequency of enriched Ala/Glu at +1 and Asp/Ile/Val at -1 around target Tyr compared phosphosite FDR between PSP and PA sets by considering positive phosphorylation evidence ("High", "Medium" or "Low" sets) in one database without taking into account any evidence in the other (SI Fig. 2, SI Table 14). The analysis revealed a generally lower FDR per each set in PA compared to the respective set in PSP, suggesting that a higher proportion of sites in the human proteome assigned to a certain phosphorylation likelihood set based on PA evidence only are true phosphosites compared to sites assigned to the same set based on only the PSP evidence (Table 2b).

\section{$\underline{\text { Functional enrichment analysis }}$}

In our analysis we categorised all 20,271 proteins in the filtered human reference proteome (SI Material 1) according to what their highest ranked Ser, Thr and Tyr site was based on phosphorylation likelihood sets in Table 1. The resulting sets (SI Table 15) were analysed in DAVID (Dennis et al., 2003) to compare functional enrichment patterns between phosphorylation likelihood sets. First, we found that across all datasets (Ser, Thr and Tyr) the protein sets containing sites ranked "High in both PSP and PA" were associated with the most significant (Benjamini-Hochberg adj. p value <0.05) functional groups (SI Fig. 3) suggesting their functional coherence i.e., sharing mappings to keywords, ontology terms or pathways. Interestingly, proteins with sites from "Low in PSP and/or PA" set as their highest ranked site and proteins which did not have any evidence phosphorylation evidence ("No evidence in PSP or PA" set) were also enriched for numerous functional categories suggesting that they too share some functional properties (SI Fig. 3). Proteins containing sites from the "Not phosphorylated" set as their highest ranked Ser/Thr/Tyr site were enriched for 1 significant functional group in the case of Tyr dataset and no functional groups in the case of Ser/Thr datasets, which was likely due to small protein sample size in those sets.

To investigate this further, we compared the top 10 enriched functional groups between the protein sets and found that proteins containing Ser, Thr and Tyr sites with most phosphorylation evidence ("High in PSP and $P A$ " set) were significantly enriched for categories and terms associated with phosphorylation such as "Phosphoprotein", "Transcription", "Nucleus" and "Alternative splicing" (Fig. 5) suggesting that those proteins were true phosphoproteins. There is a risk of generating circular evidence here, as the enriched term 
bioRxiv preprint doi: https://doi.org/10.1101/2021.04.14.439901; this version posted April 15, 2021. The copyright holder for this preprint (which was not certified by peer review) is the author/funder, who has granted bioRxiv a license to display the preprint in perpetuity. It is made available under aCC-BY 4.0 International license.

"Phosphoprotein" is a UniProt keyword, and will have been annotated based on literature evidence, potentially shared with PSP. UniProt does not yet load phosphorylation evidence from high-throughput data sets, and so classifications of phosphoproteins are generally independent of evidence used in PA. Other enriched keywords have also likely been determined based on independent evidence, and thus we believe are unbiased observations of our sets. Overall, 92.3\%, 93.9\% and 88.2\% of proteins containing Ser, Thr and Tyr sites of the highest phosphorylation likelihood respectively were enriched for the term "Phosphoprotein", which, as per description in UniProt, is a term assigned to a "protein which is post-translationally modified by the attachment of either a single phosphate group, or of a complex molecule, such as 5'-phospho-DNA, through a phosphate group" (The UniProt, 2019). Furthermore, those proteins were enriched for "Acetylation" which in some cases might indicate phosphorylation since crosstalk between acetylation and phosphorylation has been frequently reported (Espinos et al., 1999; Habibian and Ferguson, 2018). In comparison, proteins that only had sites from "Low in PSP and/or PA" set as their highest ranked Ser, Thr and Tyr sites (i.e., proteins which did not have sites with strong phosphorylation evidence) were not enriched for clear phosphorylation-associated terms and were instead enriched for categories such as "Glycoprotein", "Signal" and "Disulfide bond" and "Membrane" (Fig. 5), suggesting that the majority of those proteins were likely non-phosphoproteins and their associated phosphosites with weak evidence were therefore likely false positives. Assuming that sites with no phosphorylation evidence in PSP or PA are likely non-phosphosites (although it is possible that phosphorylation has not been investigated or localised yet), potential high FDR in the "Low in PSP and/or $P A$ " set was further supported by proteins with no phosphorylation evidence being enriched for similar functional groups (Fig. 5). In fact, we observed a clear general decrease in percentage of proteins enriched for phosphorylation-associated functional groups (where a set was enriched for at least 10 functional groups) going across our established sets suggesting an increase in phosphosite FDR across the sets (SI Fig. 4).

A

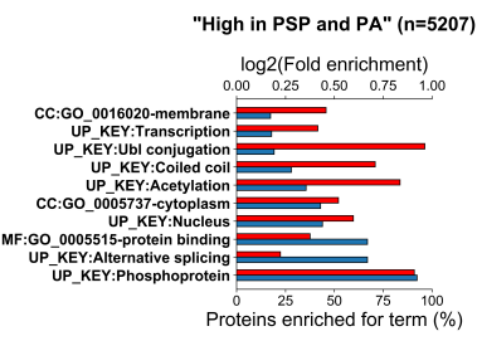

B

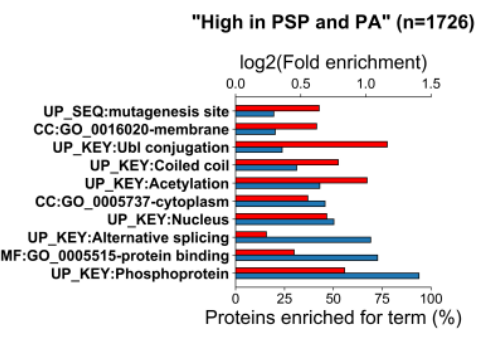

C

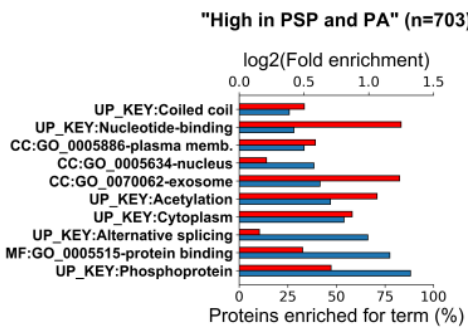

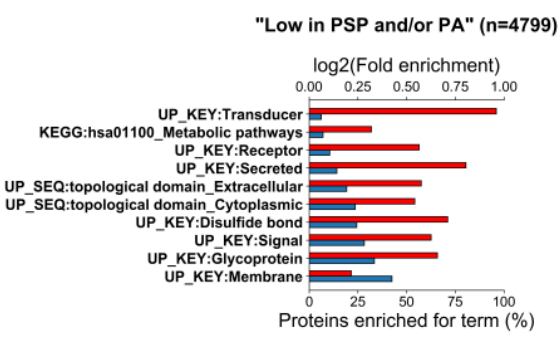
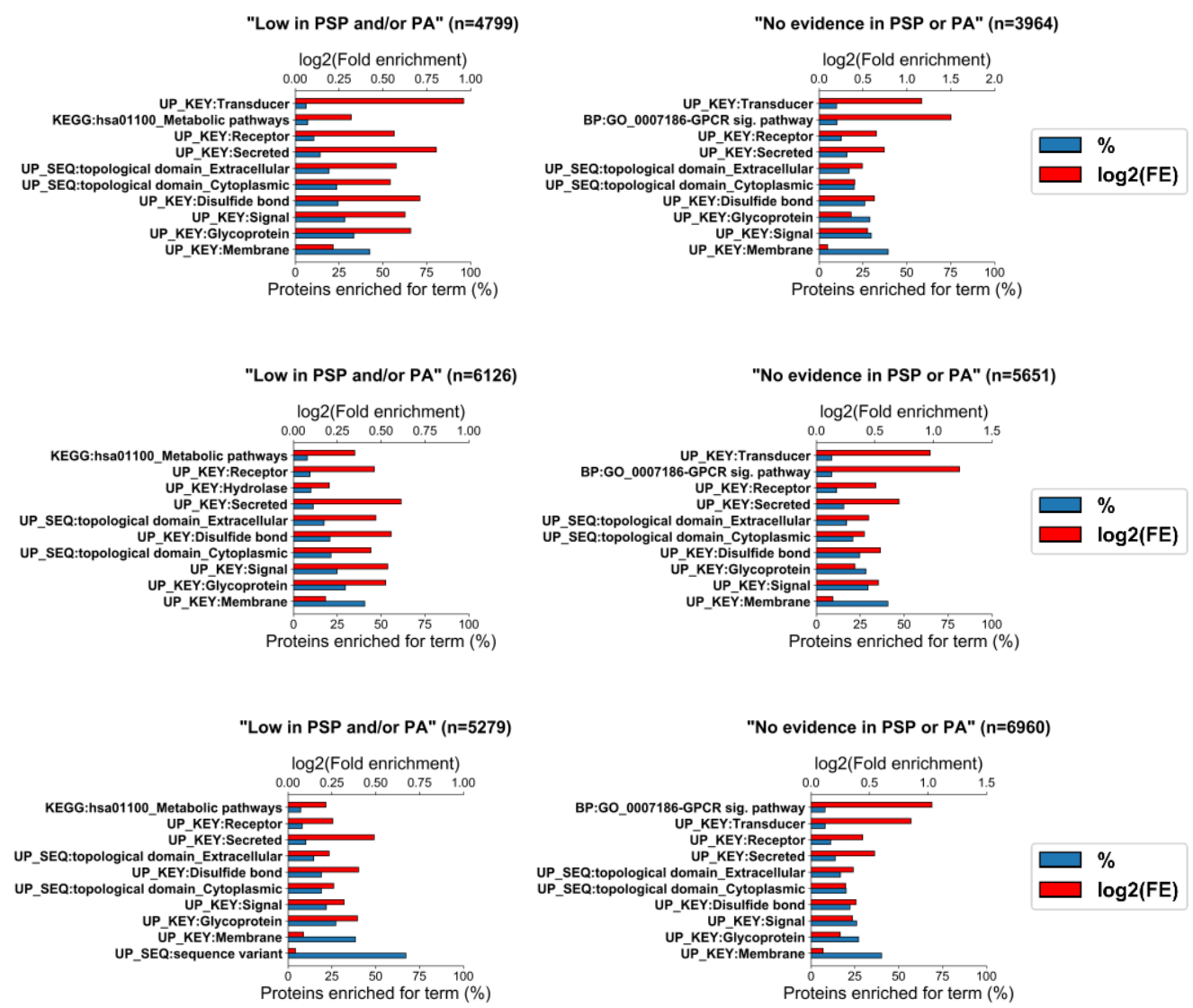

Figure 5. Top 10 functional categories for which protein sets containing various highest ranked (A) Ser, (B) Thr, (C) Tyr sites based on the amount of available phosphorylation evidence ("High in PSP and PA", "Low in PSP and/or PA", "No evidence in PSP or PA") were significantly enriched in DAVID (Benjamini-Hochberg corrected p value $<0.05$ ). For each protein set, the \% of proteins enriched for a particular functional category is given as well as the $\log 2$ (fold enrichment) for that set. The number of proteins in each set is presented by $n$. 
bioRxiv preprint doi: https://doi.org/10.1101/2021.04.14.439901; this version posted April 15, 2021. The copyright holder for this preprint (which was not certified by peer review) is the author/funder, who has granted bioRxiv a license to display the preprint in perpetuity. It is made available under aCC-BY 4.0 International license.

Our investigation of UniProt terms linked to protein sets revealed that the enrichment for term "Phosphoprotein" and other terms likely to be associated with phosphorylation ("Alternative splicing", "Nucleus", "Acetylation", "Transcription") generally decreased across confidence sets, which suggested higher FDR in sets with fewer phosphorylation evidence (Fig. 6; SI Table 16). For example, only 13.0\%, $31.7 \%$ and $36.3 \%$ of all proteins, which had Ser, Thr and Tyr sites respectively from "Low in PSP and/or PA" phosphorylation likelihood set as their most confident site, were marked as phosphoproteins in UniProt (Fig. 6, SI Table 16), suggesting that most proteins in those sets were not phosphoproteins and further highlighting that the associated sites with 1 piece of phosphorylation evidence are likely false positive identifications.
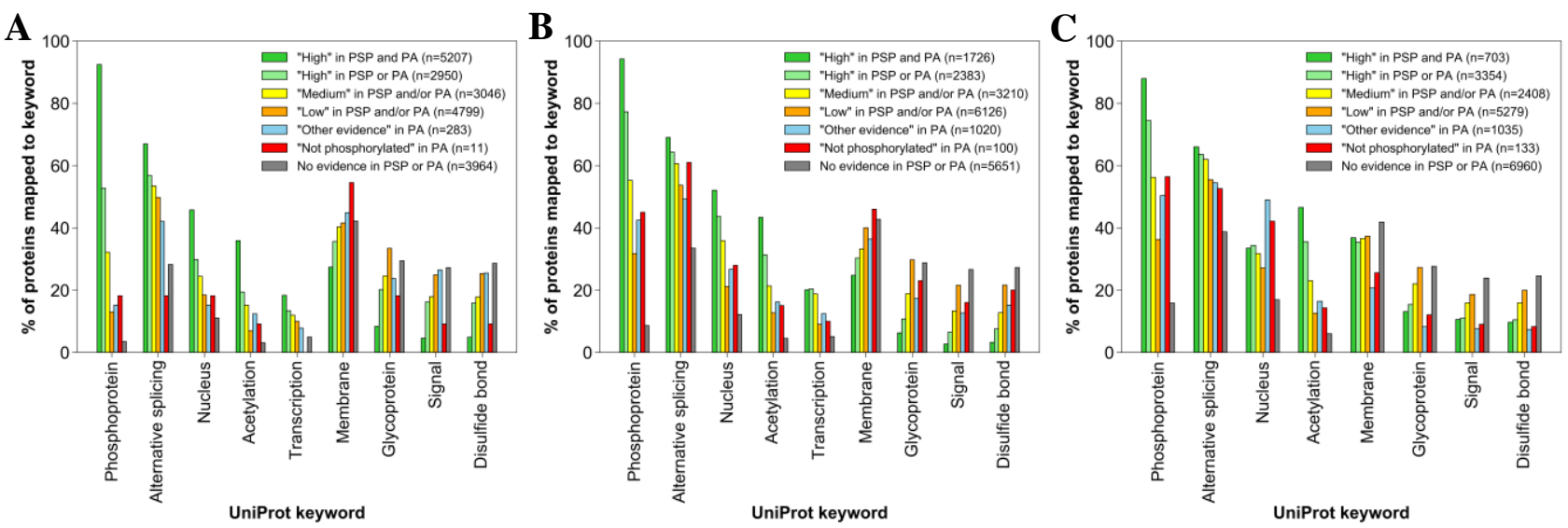

Figure 6. The percentage of proteins within sets containing (A) Ser, (B) Thr, (C) Tyr sites of various phosphorylation likelihood as their highest ranked site, annotated with specific UniProt keywords. The number of proteins in each set is presented by $n$.

\section{$\underline{\text { Secondary structure analysis }}$}

We also investigated whether Ser, Thr and Tyr sites with strong phosphorylation evidence were located more frequently within specific protein secondary structures, when compared to sites with less evidence. For example, previous analysis of thousands of phosphosites from multiple species identified hotspots within domain families of proteins, particularly near domain interfaces and adjacent to catalytic residues, where they presumably regulate enzymatic output (Beltrao et al., 2012; Strumillo et al., 2019). We found that significantly more (Fisher's test $\mathrm{p}$ value $<0.05$ ) Ser, Thr and Tyr sites with the strongest phosphorylation evidence ("High" in PSP and PA" set) were localised within coiled coils compared to sites in the "Not phosphorylated" set (Fig. 7). This might readily be explained by coiled coils being frequently found in transcription factors, the activity or subcellular location of which is often dependent on phosphorylation (Barbara et al., 2007; Baxevanis and Vinson, 1993; Pogenberg et al., 2020). Therefore, the results in Fig. 7 further indicated that there were more potential true Ser, Thr and Tyr phosphosites in "High" in PSP and PA" set compared to "Not phosphorylated" set. In terms of other analysed protein structures (beta strand, turn, alpha helix), there was no significant enrichment of sites from the highest phosphorylation confidence set within those structures when compared to the "Not phosphorylated" set (Fig. 7). In fact, our current reading of the literature suggests that it is still unclear whether phosphorylation sites are found on average to be localised more or less frequently within beta strands, turns or alpha helices, though clear evidence for localisation of PTMs at functionally important loci in proteins has been presented (Gu and Wang, 2012). 

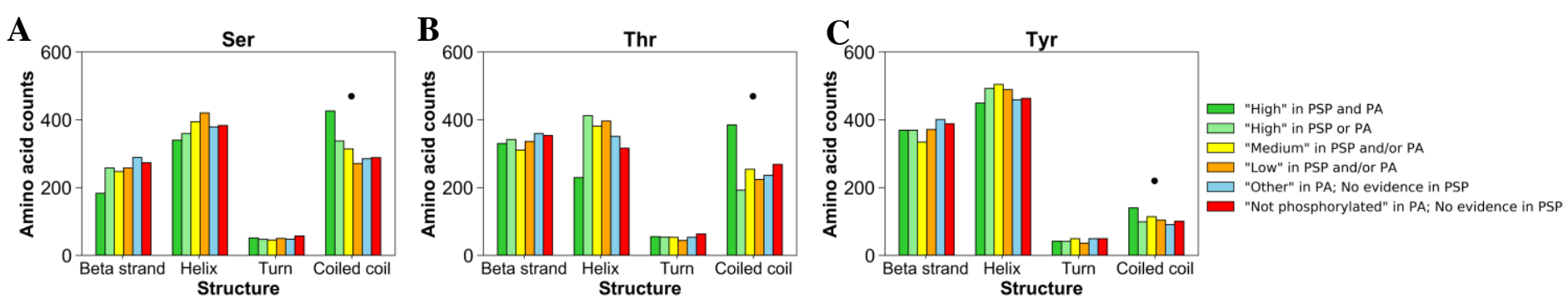

Figure 7. Normalised counts of (A) Ser; (B) Thr; (C) Tyr amino acids of various phosphorylation likelihood based on evidence in PSP and PA which are found within protein structures (beta strand, alpha helix, turn, coiled coil). Significant (Fisher's test $p$ value $<0.05$ ) enrichment of amino acids from "High" in PSP and PA" set within protein structures is highlighted by the dot symbol $(\bullet)$ when compared against the "Not phosphorylated" set.

\section{$\underline{\text { Conclusion }}$}

In our analysis we ranked all potential Ser, Thr and Tyr phosphosites in UniProt's reference human proteome according to how much quantitative and qualitative phosphorylation evidence they were assigned in PSP and PA databases. Having analysed the sites and the proteins containing them in terms of conservation, proximal site patterns, functional enrichment and structural properties, we found that Ser, Thr and Tyr sites with weak phosphosite identification evidence, particularly sites that were only observed once, were likely to be false positive identifications. This finding was further confirmed by FDR estimations across the established phosphorylation likelihood sets which revealed phosphosite FDR of $86.1 \%, 95.4 \%$ and $82.2 \%$ in sets of Ser, Thr and Tyr sites respectively where only 1 piece of identification evidence was present. Since there is a considerable presence of such sites in PSP and PA datasets, our results implied high FDR in both those datasets, although PSP was predicted to have a generally higher proportion of false positive phosphosites compared to PA. This is potentially a cause for concern since many potential false positives are presented to scientists as true phosphosites, without clear presentation of the likelihood of such claims. Nevertheless, using our FDR estimates we predicted that there are $56308 \mathrm{Ser}, 10305 \mathrm{Thr}$ and $11717 \mathrm{Tyr}$ true positive phosphosites in the human proteome that are supported by evidence in PSP and/or PA. These estimated counts are lower than other published estimates (Hornbeck et al., 2015; Ochoa et al., 2020; Safaei et al., 2011) particularly for Ser/Thr sites, presumably due to the previous inclusion of false positives and subsequent overestimation of the number of true phosphosites. We conclude that researchers must be aware of the potential for false positive sites in both public and self-generated databases and should always evaluate the evidence behind the phosphosites used in their research. We have provided here a methodological framework for estimating global FDR in largescale phosphorylation data sets, which does not rely on native scores from search engines or site localisation software. Methods for estimating global FDR in meta-analyses of phosphosites are not yet robust, and thus we would recommend that other groups similarly profile orthogonal properties of ranked sets, as we have done here, to estimate the true and false phosphosite proportions in their data. 
bioRxiv preprint doi: https://doi.org/10.1101/2021.04.14.439901; this version posted April 15, 2021. The copyright holder for this preprint (which was not certified by peer review) is the author/funder, who has granted bioRxiv a license to display the preprint in perpetuity. It is made available under aCC-BY 4.0 International license.

\section{References}

Alessi, D. R., Andjelkovic, M., Caudwell, B., Cron, P., Morrice, N., Cohen, P. and Hemmings, B. A. (1996) 'Mechanism of activation of protein kinase B by insulin and IGF-1', Embo j, 15(23), pp. 6541-51.

Altschul, S. F., Gish, W., Miller, W., Myers, E. W. and Lipman, D. J. (1990) 'Basic local alignment search tool', J Mol Biol, 215(3), pp. 403-10.

Amanchy, R., Kalume, D. E., Iwahori, A., Zhong, J. and Pandey, A. (2005) 'Phosphoproteome Analysis of HeLa Cells Using Stable Isotope Labeling with Amino Acids in Cell Culture (SILAC)', Journal of Proteome Research, 4(5), pp. 1661-1671.

Amanchy, R., Periaswamy, B., Mathivanan, S., Reddy, R., Tattikota, S. G. and Pandey, A. (2007) 'A curated compendium of phosphorylation motifs', Nature Biotechnology, 25(3), pp. 285-286.

Barbara, K. E., Willis, K. A., Haley, T. M., Deminoff, S. J. and Santangelo, G. M. (2007) 'Coiled coil structures and transcription: an analysis of the S. cerevisiae coilome', Molecular Genetics and Genomics, 278(2), pp. 135-147.

Baxevanis, A. D. and Vinson, C. R. (1993) 'Interactions of coiled coils in transcription factors: where is the specificity?', Current Opinion in Genetics \& Development, 3(2), pp. 278-285.

Beausoleil, S. A., Villén, J., Gerber, S. A., Rush, J. and Gygi, S. P. (2006) 'A probability-based approach for high-throughput protein phosphorylation analysis and site localization', Nature Biotechnology, 24(10), pp. 1285-1292.

Beltrao, P., Albanèse, V., Kenner, L. R., Swaney, D. L., Burlingame, A., Villén, J., Lim, W. A., Fraser, J. S., Frydman, J. and Krogan, N. J. (2012) 'Systematic functional prioritization of protein posttranslational modifications', Cell, 150(2), pp. 413-25.

Boekhorst, J., van Breukelen, B., Heck, A., Jr. and Snel, B. (2008) 'Comparative phosphoproteomics reveals evolutionary and functional conservation of phosphorylation across eukaryotes', Genome biology, 9(10), pp. R144-R144.

Byrne, D. P., Clarke, C. J., Brownridge, P. J., Kalyuzhnyy, A., Perkins, S., Campbell, A., Mason, D., Jones, A. R., Eyers, P. A. and Eyers, C. E. (2020) 'Use of the Polo-like kinase 4 (PLK4) inhibitor centrinone to investigate intracellular signalling networks using SILAC-based phosphoproteomics', Biochem J, 477(13), pp. 2451-2475.

Caron, D., Byrne, D. P., Thebault, P., Soulet, D., Landry, C. R., Eyers, P. A. and Elowe, S. (2016) 'Mitotic phosphotyrosine network analysis reveals that tyrosine phosphorylation regulates Polo-like kinase 1 (PLK1)', Sci Signal, 9(458), pp. rs14.

Chalkley, R. J. and Clauser, K. R. (2012) 'Modification site localization scoring: strategies and performance', Mol Cell Proteomics, 11(5), pp. 3-14.

Chen, S. C.-C., Chen, F.-C. and Li, W.-H. (2010) 'Phosphorylated and nonphosphorylated serine and threonine residues evolve at different rates in mammals', Molecular biology and evolution, 27(11), pp. 2548-2554.

Cohen, P. (2001) 'The role of protein phosphorylation in human health and disease. The Sir Hans Krebs Medal Lecture', European journal of biochemistry / FEBS, 268, pp. 5001-10.

Cohen, P. (2002) 'The origins of protein phosphorylation', Nature Cell Biology, 4(5), pp. E127-E130.

Cox, J., Neuhauser, N., Michalski, A., Scheltema, R. A., Olsen, J. V. and Mann, M. (2011) 'Andromeda: a peptide search engine integrated into the MaxQuant environment', J Proteome Res, 10(4), pp. 1794-805.

Dennis, G., Sherman, B. T., Hosack, D. A., Yang, J., Gao, W., Lane, H. C. and Lempicki, R. A. (2003) 'DAVID: Database for Annotation, Visualization, and Integrated Discovery', Genome Biology, 4(5), pp. P3.

Dephoure, N., Gould, K. L., Gygi, S. P. and Kellogg, D. R. (2013) 'Mapping and analysis of phosphorylation sites: a quick guide for cell biologists', Molecular biology of the cell, 24(5), pp. 535-542.

Desiere, F., Deutsch, E. W., King, N. L., Nesvizhskii, A. I., Mallick, P., Eng, J., Chen, S., Eddes, J., Loevenich, S. N. and Aebersold, R. (2006) 'The PeptideAtlas project', Nucleic acids research, 34(Database issue), pp. D655-D658. 
bioRxiv preprint doi: https://doi.org/10.1101/2021.04.14.439901; this version posted April 15, 2021. The copyright holder for this preprint (which was not certified by peer review) is the author/funder, who has granted bioRxiv a license to display the preprint in perpetuity. It is made available under aCC-BY 4.0 International license.

Deutsch, E. W., Mendoza, L., Shteynberg, D., Farrah, T., Lam, H., Tasman, N., Sun, Z., Nilsson, E., Pratt, B., Prazen, B., Eng, J. K., Martin, D. B., Nesvizhskii, A. I. and Aebersold, R. (2010) 'A guided tour of the TransProteomic Pipeline', Proteomics, 10(6), pp. 1150-1159.

Dinkel, H., Chica, C., Via, A., Gould, C. M., Jensen, L. J., Gibson, T. J. and Diella, F. (2011) 'Phospho.ELM: a database of phosphorylation sites--update 2011', Nucleic acids research, 39(Database issue), pp. D261-D267.

Edgar, R. C. (2004) 'MUSCLE: multiple sequence alignment with high accuracy and high throughput', Nucleic acids research, 32(5), pp. 1792-1797.

Elias, J. E. and Gygi, S. P. (2007) 'Target-decoy search strategy for increased confidence in large-scale protein identifications by mass spectrometry', Nature Methods, 4(3), pp. 207-214.

Espinos, E., Le Van Thaï, A., Pomiès, C. and Weber, M. J. (1999) 'Cooperation between phosphorylation and acetylation processes in transcriptional control', Mol Cell Biol, 19(5), pp. 3474-84.

Ferries, S., Perkins, S., Brownridge, P. J., Campbell, A., Eyers, P. A., Jones, A. R. and Eyers, C. E. (2017) 'Evaluation of Parameters for Confident Phosphorylation Site Localization Using an Orbitrap Fusion Tribrid Mass Spectrometer', J Proteome Res, 16(9), pp. 3448-3459.

Fisher, R. A. (1934) Statistical methods for research workers. Edinburgh: Oliver and Boyd.

Gnad, F., Gunawardena, J. and Mann, M. (2011) 'PHOSIDA 2011: the posttranslational modification database', Nucleic acids research, 39(Database issue), pp. D253-D260.

Goedert, M., Spillantini, M. G., Cairns, N. J. and Crowther, R. A. (1992) 'Tau proteins of alzheimer paired helical filaments: Abnormal phosphorylation of all six brain isoforms', Neuron, 8(1), pp. 159-168.

Gu, G. M. and Wang, J. K. (2012) '[DNA-binding profiles of mammalian transcription factors]', Yi Chuan, 34(8), pp. 950-68.

Habibian, J. and Ferguson, B. S. (2018) 'The Crosstalk between Acetylation and Phosphorylation: Emerging New Roles for HDAC Inhibitors in the Heart', Int J Mol Sci, 20(1).

Hall, F. L. and Vulliet, P. R. (1991) 'Proline-directed protein phosphorylation and cell cycle regulation', Current Opinion in Cell Biology, 3(2), pp. 176-184.

Hardman, G., Perkins, S., Brownridge, P. J., Clarke, C. J., Byrne, D. P., Campbell, A. E., Kalyuzhnyy, A., Myall, A., Eyers, P. A., Jones, A. R. and Eyers, C. E. (2019) 'Strong anion exchange-mediated phosphoproteomics reveals extensive human non-canonical phosphorylation', The EMBO journal, 38(21), pp. e100847-e100847.

Hornbeck, P. V., Zhang, B., Murray, B., Kornhauser, J. M., Latham, V. and Skrzypek, E. (2015) 'PhosphoSitePlus, 2014: mutations, PTMs and recalibrations', Nucleic acids research, 43(Database issue), pp. D512-D520.

Hutti, J. E., Jarrell, E. T., Chang, J. D., Abbott, D. W., Storz, P., Toker, A., Cantley, L. C. and Turk, B. E. (2004) 'A rapid method for determining protein kinase phosphorylation specificity', Nat Methods, 1(1), pp. 27 9.

Johnson, L. N., Lowe, E. D., Noble, M. E. and Owen, D. J. (1998) 'The Eleventh Datta Lecture. The structural basis for substrate recognition and control by protein kinases', FEBS Lett, 430(1-2), pp. 1-11.

Käll, L., Storey, J. D. and Noble, W. S. (2009) 'QVALITY: non-parametric estimation of q-values and posterior error probabilities', Bioinformatics (Oxford, England), 25(7), pp. 964-966.

Keshwani, M. M., Aubol, B. E., Fattet, L., Ma, C.-T., Qiu, J., Jennings, P. A., Fu, X.-D. and Adams, J. A. (2015) 'Conserved proline-directed phosphorylation regulates SR protein conformation and splicing function', The Biochemical journal, 466(2), pp. 311-322.

Kettenbach, A. N., Wang, T., Faherty, B. K., Madden, D. R., Knapp, S., Bailey-Kellogg, C. and Gerber, S. A. (2012) 'Rapid determination of multiple linear kinase substrate motifs by mass spectrometry', Chem Biol, 19(5), pp. 608-18.

Landry, C. R., Levy, E. D. and Michnick, S. W. (2009) 'Weak functional constraints on phosphoproteomes', Trends in Genetics, 25(5), pp. 193-197. 
bioRxiv preprint doi: https://doi.org/10.1101/2021.04.14.439901; this version posted April 15, 2021. The copyright holder for this preprint (which was not certified by peer review) is the author/funder, who has granted bioRxiv a license to display the preprint in perpetuity. It is made available under aCC-BY 4.0 International license.

Lee, D. C. H., Jones, A. R. and Hubbard, S. J. (2015) 'Computational phosphoproteomics: from identification to localization', Proteomics, 15(5-6), pp. 950-963.

Lienhard, G. E. (2008) 'Non-functional phosphorylations?', Trends in Biochemical Sciences, 33(8), pp. 351352.

Lu, K. P., Liou, Y.-C. and Zhou, X. Z. (2002) 'Pinning down proline-directed phosphorylation signaling', Trends in Cell Biology, 12(4), pp. 164-172.

Malik, R., Nigg, E. A. and Körner, R. (2008) 'Comparative conservation analysis of the human mitotic phosphoproteome', Bioinformatics, 24(12), pp. 1426-1432.

Nousiainen, M., Silljé, H. H. W., Sauer, G., Nigg, E. A. and Körner, R. (2006) 'Phosphoproteome analysis of the human mitotic spindle', Proceedings of the National Academy of Sciences, 103(14), pp. 5391.

Ochoa, D., Jarnuczak, A. F., Viéitez, C., Gehre, M., Soucheray, M., Mateus, A., Kleefeldt, A. A., Hill, A., Garcia-Alonso, L., Stein, F., Krogan, N. J., Savitski, M. M., Swaney, D. L., Vizcaíno, J. A., Noh, K.-M. and Beltrao, P. (2020) 'The functional landscape of the human phosphoproteome', Nature Biotechnology, 38(3), pp. 365-373.

Olsen, J., Blagoev, B., Gnad, F., Macek, B., Kumar, C., Mortensen, P. and Mann, M. (2006) 'Global, In Vivo, and Site-Specific Phosphorylation Dynamics in Signaling Networks', Cell, 127, pp. 635-48.

Olsen, J. V., Vermeulen, M., Santamaria, A., Kumar, C., Miller, M. L., Jensen, L. J., Gnad, F., Cox, J., Jensen, T. S., Nigg, E. A., Brunak, S. and Mann, M. (2010) 'Quantitative Phosphoproteomics Reveals Widespread Full Phosphorylation Site Occupancy During Mitosis', Science Signaling, 3(104), pp. ra3.

Pietrangelo, A. and Ridgway, N. D. (2019) 'Phosphorylation of a serine/proline-rich motif in oxysterol binding protein-related protein $4 \mathrm{~L}(\mathrm{ORP} 4 \mathrm{~L})$ regulates cholesterol and vimentin binding', PloS one, 14(3), pp. e0214768-e0214768.

Pogenberg, V., Ballesteros-Álvarez, J., Schober, R., Sigvaldadóttir, I., Obarska-Kosinska, A., Milewski, M., Schindl, R., Ögmundsdóttir, M. H., Steingrímsson, E. and Wilmanns, M. (2020) 'Mechanism of conditional partner selectivity in MITF/TFE family transcription factors with a conserved coiled coil stammer motif', Nucleic Acids Research, 48(2), pp. 934-948.

Rikova, K., Guo, A., Zeng, Q., Possemato, A., Yu, J., Haack, H., Nardone, J., Lee, K., Reeves, C., Li, Y., Hu, Y., Tan, Z., Stokes, M., Sullivan, L., Mitchell, J., Wetzel, R., Macneill, J., Ren, J. M., Yuan, J., Bakalarski, C. E., Villen, J., Kornhauser, J. M., Smith, B., Li, D., Zhou, X., Gygi, S. P., Gu, T. L., Polakiewicz, R. D., Rush, J. and Comb, M. J. (2007) 'Global survey of phosphotyrosine signaling identifies oncogenic kinases in lung cancer', Cell, 131(6), pp. 1190-203.

Rush, J., Moritz, A., Lee, K. A., Guo, A., Goss, V. L., Spek, E. J., Zhang, H., Zha, X. M., Polakiewicz, R. D. and Comb, M. J. (2005) 'Immunoaffinity profiling of tyrosine phosphorylation in cancer cells', Nat Biotechnol, 23(1), pp. 94-101.

Safaei, J., Maňuch, J., Gupta, A., Stacho, L. and Pelech, S. (2011) 'Prediction of 492 human protein kinase substrate specificities', Proteome Science, 9(1), pp. S6.

Sharma, K., D'Souza, R. C., Tyanova, S., Schaab, C., Wiśniewski, J. R., Cox, J. and Mann, M. (2014) 'Ultradeep human phosphoproteome reveals a distinct regulatory nature of Tyr and Ser/Thr-based signaling', Cell Rep, 8(5), pp. 1583-94.

Shteynberg, D. D., Deutsch, E. W., Campbell, D. S., Hoopmann, M. R., Kusebauch, U., Lee, D., Mendoza, L., Midha, M. K., Sun, Z., Whetton, A. D. and Moritz, R. L. (2019) 'PTMProphet: Fast and Accurate Mass Modification Localization for the Trans-Proteomic Pipeline', Journal of proteome research, 18(12), pp. 42624272.

Söderholm, S., Hintsanen, P., Öhman, T., Aittokallio, T. and Nyman, T. A. (2014) 'PhosFox: a bioinformatics tool for peptide-level processing of LC-MS/MS-based phosphoproteomic data', Proteome science, 12, pp. 3636.

Songyang, Z., Blechner, S., Hoagland, N., Hoekstra, M. F., Piwnica-Worms, H. and Cantley, L. C. (1994) 'Use of an oriented peptide library to determine the optimal substrates of protein kinases', Curr Biol, 4(11), pp. 97382. 
Songyang, Z., Lu, K. P., Kwon, Y. T., Tsai, L. H., Filhol, O., Cochet, C., Brickey, D. A., Soderling, T. R., Bartleson, C., Graves, D. J., DeMaggio, A. J., Hoekstra, M. F., Blenis, J., Hunter, T. and Cantley, L. C. (1996) 'A structural basis for substrate specificities of protein Ser/Thr kinases: primary sequence preference of casein kinases I and II, NIMA, phosphorylase kinase, calmodulin-dependent kinase II, CDK5, and Erk1', Molecular and cellular biology, 16(11), pp. 6486-6493.

Strumillo, M. J., Oplová, M., Viéitez, C., Ochoa, D., Shahraz, M., Busby, B. P., Sopko, R., Studer, R. A., Perrimon, N., Panse, V. G. and Beltrao, P. (2019) 'Conserved phosphorylation hotspots in eukaryotic protein domain families', Nat Commun, 10(1), pp. 1977.

Studer, R. A., Rodriguez-Mias, R. A., Haas, K. M., Hsu, J. I., Viéitez, C., Solé, C., Swaney, D. L., Stanford, L. B., Liachko, I., Böttcher, R., Dunham, M. J., de Nadal, E., Posas, F., Beltrao, P. and Villén, J. (2016) 'Evolution of protein phosphorylation across 18 fungal species', Science, 354(6309), pp. 229-232.

Sugiyama, N., Imamura, H. and Ishihama, Y. (2019) 'Large-scale Discovery of Substrates of the Human Kinome', Scientific Reports, 9(1), pp. 10503.

Taus, T., Köcher, T., Pichler, P., Paschke, C., Schmidt, A., Henrich, C. and Mechtler, K. (2011) 'Universal and confident phosphorylation site localization using phosphoRS', J Proteome Res, 10(12), pp. 5354-62.

The UniProt, C. (2019) 'UniProt: a worldwide hub of protein knowledge', Nucleic Acids Research, 47(D1), pp. D506-D515.

Virtanen, P. and Gommers, R. and Oliphant, T. E. and Haberland, M. and Reddy, T. and Cournapeau, D. and Burovski, E. and Peterson, P. and Weckesser, W. and Bright, J. and van der Walt, S. J. and Brett, M. and Wilson, J. and Millman, K. J. and Mayorov, N. and Nelson, A. R. J. and Jones, E. and Kern, R. and Larson, E. and Carey, C. J. and Polat, I. and Feng, Y. and Moore, E. W. and VanderPlas, J. and Laxalde, D. and Perktold, J. and Cimrman, R. and Henriksen, I. and Quintero, E. A. and Harris, C. R. and Archibald, A. M. and Ribeiro, A. H. and Pedregosa, F. and van Mulbregt, P. and Vijaykumar, A. and Bardelli, A. P. and Rothberg, A. and Hilboll, A. and Kloeckner, A. and Scopatz, A. and Lee, A. and Rokem, A. and Woods, C. N. and Fulton, C. and Masson, C. and Häggström, C. and Fitzgerald, C. and Nicholson, D. A. and Hagen, D. R. and Pasechnik, D. V. and Olivetti, E. and Martin, E. and Wieser, E. and Silva, F. and Lenders, F. and Wilhelm, F. and Young, G. and Price, G. A. and Ingold, G.-L. and Allen, G. E. and Lee, G. R. and Audren, H. and Probst, I. and Dietrich, J. P. and Silterra, J. and Webber, J. T. and Slavič, J. and Nothman, J. and Buchner, J. and Kulick, J. and Schönberger, J. L. and de Miranda Cardoso, J. V. and Reimer, J. and Harrington, J. and Rodríguez, J. L. C. and Nunez-Iglesias, J. and Kuczynski, J. and Tritz, K. and Thoma, M. and Newville, M. and Kümmerer, M. and Bolingbroke, M. and Tartre, M. and Pak, M. and Smith, N. J. and Nowaczyk, N. and Shebanov, N. and Pavlyk, O. and Brodtkorb, P. A. and Lee, P. and McGibbon, R. T. and Feldbauer, R. and Lewis, S. and Tygier, S. and Sievert, S. and Vigna, S. and Peterson, S. and More, S. and Pudlik, T. and Oshima, T. and Pingel, T. J. and Robitaille, T. P. and Spura, T. and Jones, T. R. and Cera, T. and Leslie, T. and Zito, T. and Krauss, T. and Upadhyay, U. and Halchenko, Y. O. and Vázquez-Baeza, Y. and SciPy, C. (2020) 'SciPy 1.0: fundamental algorithms for scientific computing in Python', Nature Methods, 17(3), pp. 261-272.

Wälchli, S., Espanel, X., Harrenga, A., Rossi, M., Cesareni, G. and Hooft van Huijsduijnen, R. (2004) 'Probing protein-tyrosine phosphatase substrate specificity using a phosphotyrosine-containing phage library', $J$ Biol Chem, 279(1), pp. 311-8.

Wettenhall, R. E., Aebersold, R. H. and Hood, L. E. (1991) 'Solid-phase sequencing of 32P-labeled phosphopeptides at picomole and subpicomole levels', Methods Enzymol, 201, pp. 186-99.

Wettenhall, R. E., Erikson, E. and Maller, J. L. (1992) 'Ordered multisite phosphorylation of Xenopus ribosomal protein S6 by S6 kinase II', J Biol Chem, 267(13), pp. 9021-7.

Zahn-Zabal, M., Michel, P.-A., Gateau, A., Nikitin, F., Schaeffer, M., Audot, E., Gaudet, P., Duek, P. D., Teixeira, D., Rech de Laval, V., Samarasinghe, K., Bairoch, A. and Lane, L. (2020) 'The neXtProt knowledgebase in 2020: data, tools and usability improvements', Nucleic Acids Research, 48(D1), pp. D328D334. 